\title{
An analysis of equivalent operator preconditioning for equation-free Newton-Krylov methods
}

Giovanni Samaey

Wim Vanroose

Report TW 537, March 2009

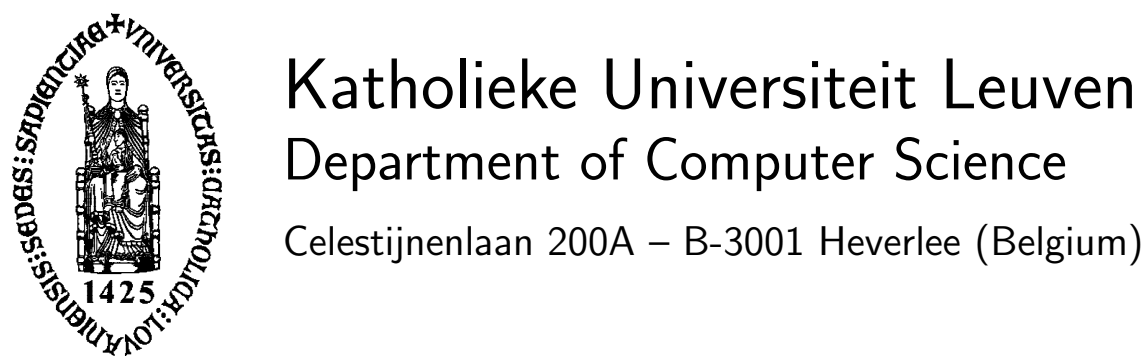




\title{
An analysis of equivalent operator preconditioning for equation-free Newton-Krylov methods
}

\author{
Giovanni Samaey* \\ Wim Vanroose ${ }^{\dagger}$ \\ Report TW 537, March 2009 \\ Department of Computer Science, K.U.Leuven
}

\begin{abstract}
We consider the computation of a fixed point of a time-stepper using NewtonKrylov methods, and propose and analyze equivalent operator preconditioning for the resulting linear systems. For a linear, scalar advection-reaction-diffusion equation, we investigate in detail how the convergence rate depends on the choice of preconditioner parameters and on the time discretization. The results are especially valuable when computing fixed points of a coarse time-stepper in the equation-free multiscale framework, in which one simulates an unavailable coarse-scale model by wrapping a set of computational routines around appropriately initialized fine-scale simulations. Both analytical results and numerical experiments are presented, showing that one can speed up the convergence of iterative methods significantly for a wide range of parameter values in the preconditioner.
\end{abstract}

\footnotetext{
${ }^{*}$ Department of Computer Science, K.U. Leuven, Celestijnenlaan 200A, 3001 Leuven, Belgium

${ }^{\dagger}$ Department of Mathematics and Computer Science, Universiteit Antwerpen, Middelheimlaan 1, 2020 Antwerpen, Belgium
} 


\section{Introduction}

We consider systems for which only a time-stepper (and not the corresponding partial differential equation) is available, and one is interested in the direct computation of fixed points. This situation arises when dealing with legacy codes, and also in the context of equation-free multiscale computation, as will be discussed below. Several classes of methods exist that only use selected matrix-vector products with the system's Jacobian, estimated using repeated calls to the time-stepper starting from several nearby initial conditions. We mention the recursive projection method (RPM) [27] and generalizations [18, 19], and Jacobian-free Newton-Krylov methods. For the latter class, efficiency depends on the use of a good preconditioner for the linear systems arising in each Newton iteration; see e.g. [15] for an review.

In this paper, we investigate equivalent operator preconditioning for the Newton-Krylov computation of fixed points of a time-stepper for an unavailable reaction-advection-diffusion equation

$$
\partial_{t} U=\mathcal{A} U=D \partial_{x}^{2} U+\beta \partial_{x} U+\delta U,
$$

in which the coefficients $D, \beta$ and $\delta$ are unknown in closed form. As a preconditioner, we propose to use a time-stepper for an arbitrary reaction-advection-diffusion equation

$$
\partial_{t} U=\mathcal{B} U=D^{*} \partial_{x}^{2} U+\beta^{*} \partial_{x} U+\delta^{*} U,
$$

in which the coefficients $D^{*}, \beta^{*}$ and $\delta^{*}$ are chosen to optimize the convergence rate.

For elliptic operators, preconditioning using a different, simpler operator has been developed in a large number of works, starting with the early papers [3, 12], and later e.g. $[2,20,4,31,28]$. The notion of an equivalent operator has been used to construct a general theory for such preconditioning, see e.g. [8, 20, 21, 11]; for operators that are a compact perturbation of unity, a superlinear convergence result has been obtained [1]. In [20], an analysis of so-called optimal equivalent preconditioners was presented for general second-order elliptic problems in two space dimensions; the main idea is to precondition using the leading part $L$ plus a zeroth-order term $\delta^{*} I$, in which $\delta^{*}$ is chosen to optimize the convergence factor. If one can obtain small convergence factors, this is an appealing strategy, since the preconditioner $L+\delta^{*} I$ is symmetric, positive definite, and easily inverted, especially for large $\delta^{*}$. We note that a generalization of this idea, in which $\delta^{*}$ is allowed to be complex, has also been studied to precondition Helmholtz equations [7].

One particular situation in which this analysis is useful, is the computation of coarse fixed points in the equation-free framework [13, 14]. This framework considers systems for which only a fine-scale description is available, and a corresponding coarse-scale model (such as a reaction-advection-diffusion PDE) should conceptually exist, but is difficult (or impossible) to derive analytically from the underlying fine-scale model without introducing simplifying assumptions that are hard to justify. Typical cases include bacterial chemotaxis 
[6] and the ionization reaction in gases [17]. The key idea, which was first proposed in [29], is to construct a coarse time-stepper for the unavailable coarse equation as a three step procedure: (1) lifting, i.e. the creation of appropriate initial conditions for the fine-scale model, conditioned upon the coarse state at time $t^{*} ;(2)$ simulation, using the fine-scale model, over the time interval $\left[t^{*}, t^{*}+\Delta t\right]$; and (3) restriction, i.e. the extraction of the coarse state at time $t^{*}+\Delta t$. In the equation-free context, the Jacobian of the coarse timestepper is not explicitly available, and therefore one cannot use preconditioning techniques that rely on algebraic manipulation, such as incomplete LU factorization (ILU). It has been proposed to construct a preconditioner based on a time-stepper for an approximate coarse model, e.g. obtained from a mean field approximation or an asymptotic expansion $[22,26]$. By preconditioning with an equivalent operator, one avoids the derivation of an approximate macroscopic model, since only the order of the coarse equation is required. This paper exclusively deals with the analysis of equivalent operator preconditioning for linear scalar problems with constant coefficients. For an equation-free numerical study of coarse systems of non-linear equations with space-dependent coefficients, we refer to [25], where we use equivalent operator preconditioners to compute traveling waves of a fine-scale multi-speed lattice Boltzmann problem.

The remainder of this paper is organized as follows. We briefly review the basic properties of the coarse time-stepper and its use in an equation-free Newton-Krylov method in section 2. In section 3 , we describe the preconditioning procedure in detail, and summarize some results that are available in the literature on the choice of an optimal equivalent operator. We analyze the spectrum of the preconditioned system as a function of the preconditioning parameters, both in the continuous (section 4) and the discrete case (section 5). An additional complication in the discrete case is the effect of the time discretization. In the linear scalar case that is considered in this paper, the preconditioner $\mathcal{B}$ becomes identical to $\mathcal{A}$ for the correct choice of parameters, provided the same space and time discretization is used for both operators. The analysis reveals to what extent the preconditioned spectrum is affected by an incomplete knowledge of the parameters of $\mathcal{A}$. In section 6 , we present numerical convergence tests for the Richardson iteration and for GMRES. These results show that, besides the spectral convergence factor, issues related to non-normality (and the associated stagnation of iterative methods) should be taken into account when choosing the preconditioning parameters. We conclude in section 7 with a brief discussion on the applicability of the results. 


\section{Equation-free Newton-Krylov methods}

\subsection{The coarse time-stepper}

We briefly review the coarse time-stepper as it was introduced by Kevrekidis et al. [13]. Consider an abstract fine-scale evolution law, and corresponding time-stepper,

$$
\partial_{t} u(\mathbf{x}, t)=f(u(\mathbf{x}, t)), \quad u(\mathbf{x}, t+\delta t)=s(u(\mathbf{x}, t), \delta t),
$$

in which $u(\mathbf{x}, t)$ represent the fine-scale state variables, $\mathbf{x} \in D_{m}$ and $t$ are the fine-scale independent variables, $\partial_{t}$ denotes the time derivative, and $\delta t$ is the size of the fine-scale time-step. We assume that a coarse model, denoted by

$$
\partial_{t} U(\mathbf{X}, t)=F(U(\mathbf{X}, t))
$$

conceptually exists, but is unavailable in closed form. In equation (2.2), $U(\mathbf{X}, t)$ represent the coarse state variables, and $\mathbf{X} \in D_{M}$ and $t$ are the coarse independent variables. The aim is to obtain a coarse time-stepper $\bar{S}$ for the variables $U(\mathbf{X}, t)$ as

$$
\bar{U}(\mathbf{X}, t+\Delta t)=\bar{S}(\bar{U}(\mathbf{X}, t), \Delta t)
$$

where $\Delta t$ denotes the size of the coarse time-step, and the bars have been introduced to emphasize the fact that the time-stepper for the coarse variables is an approximation of a time-stepper for (2.2), since this equation is not explicitly known.

To this end, we introduce two operators that make the transition between fine-scale and coarse variables. We define a lifting operator,

$$
\mu: U(\mathbf{X}, t) \mapsto u(\mathbf{x}, t)=\mu(U(\mathbf{X}, t)),
$$

which maps coarse to fine-scale variables, and its complement, the restriction operator,

$$
\mathcal{M}: u(\mathbf{x}, t) \mapsto U(\mathbf{X}, t)=\mathcal{M}(u(\mathbf{x}, t)) .
$$

The restriction operator can often be determined as soon as the coarse variables are known. For instance, when the fine-scale model consists of an evolving ensemble of many particles, the restriction typically consists of the computation of the first few low order moments of the distribution (density, momentum, energy).

The construction of the lifting operator is usually more involved. Again taking the example of a particle model, we need to define a mapping from a few low order moments to initial conditions for each of the particles. The assumption that an equation exists and closes at the level of these low order moments, implies that the higher order moments become functionals of the low order ones on time-scales that are fast compared to the overall system evolution (slaving); but unfortunately, these slaving relations are unknown 
(since the coarse evolution law is also unknown). Several approaches have been suggested to address this problem. We refer to $[9,10,30]$ for an algorithm that only uses a time-stepper for the original fine-scale system.

Given an initial condition for the coarse variables $U\left(\mathbf{X}, t^{*}\right)$ at some time $t^{*}$, we construct the time-stepper (2.3) in the following way:

1. Lifting. Using the lifting operator (2.4), create appropriate initial conditions $u\left(\mathbf{x}, t^{*}\right)$ for the fine-scale time-stepper (2.1), consistent with the coarse variables.

2. Simulation. Use the fine-scale time-stepper (2.1) to compute the fine-scale state $u(\mathbf{x}, t)$ for $t \in\left[t^{*}, t^{*}+\Delta t\right]$.

3. Restriction. Obtain the coarse state $U\left(\mathbf{X}, t^{*}+\Delta t\right)$ from the fine-scale state $u\left(\mathbf{x}, t^{*}+\right.$ $\Delta t$ ) using the restriction operator (2.5).

Assuming $\Delta t=k \delta t$, this can be written as

$$
\bar{U}(\mathbf{X}, t+\Delta t)=\bar{S}(\bar{U}(\mathbf{X}, t), \Delta t)=\mathcal{M}\left(s^{k}(\mu(\bar{U}(\mathbf{X}, t)), \delta t)\right),
$$

where we have represented the $k$ fine-scale time-steps by a superscript on $s$. If the finescale model is stochastic, one may need to perform multiple replica simulations, using an ensemble of fine-scale initial conditions, to obtain an accurate result with a sufficiently low variance.

\subsection{Computation of coarse steady states}

A coarse steady state can be computed as a fixed point of the coarse time-stepper,

$$
\bar{G}(\bar{U} ; \Delta t)=\bar{U}-\bar{S}(\bar{U}, \Delta t)=0 .
$$

This nonlinear system can be solved with a Newton-Raphson procedure,

$$
\bar{U}^{(k+1)}=\bar{U}^{(k)}+d \bar{U}^{(k)}
$$

with the correction $d \bar{U}^{(k)}$ the solution of the linear system

$$
\bar{G}_{U}\left(\bar{U}^{(k)}, \Delta t\right) d \bar{U}^{(k)}=\left(I-\bar{S}_{U}\left(\bar{U}^{(k)}, \Delta t\right)\right) d \bar{U}^{(k)}=-G\left(\bar{U}^{(k)}, \Delta t\right),
$$

where $\bar{G}_{U}\left(\bar{U}^{(k)}, \Delta t\right)$ and $\bar{S}_{U}\left(\bar{U}^{(k)}, \Delta t\right)$ denote the linearization of resp. $\bar{G}(\bar{U}, \Delta t)$ and $\bar{S}(\bar{U}, \Delta t)$ around the point $\bar{U}^{(k)}$. Since $\bar{S}_{U}\left(\bar{U}^{(k)}, \Delta t\right)$ is the linearization of a coarse time-stepper, we do not have its explicit formula. However, we can estimate matrix-vector products as

$$
\left(I-\bar{S}_{U}(\bar{U}, \Delta t)\right) v \approx v-\frac{\bar{S}(\bar{U}+\epsilon v, \Delta t)-\bar{S}(\bar{U}, \Delta t)}{\epsilon} .
$$

Therefore, we solve the linear system (2.9) using a Krylov method, such as GMRES [24]. 
The convergence rate of GMRES depends heavily on the spectral properties of the system matrix in equation (2.9). For GMRES to converge rapidly, the eigenvalues should be clustered, e.g. around one [23]. Since $\bar{S}(\bar{U}, \Delta t)$ is a time-stepper, most of its eigenvalues are contained within the unit circle; the eigenvalues of $\bar{G}_{U}(\bar{U}, \Delta t)$ can then lie arbitrarily close to zero. Preconditioning will be therefore be necessary to bound the eigenvalues away from zero while keeping the large eigenvalues small.

We define a preconditioning matrix $M(\bar{U}, \Delta t)$, and replace the linear system $(2.9)$ with

$$
M\left(\bar{U}^{(k)}, \Delta t\right)^{-1} \bar{G}_{U}\left(\bar{U}^{(k)}, \Delta t\right) d \bar{U}^{(k)}=-M\left(\bar{U}^{(k)}, \Delta t\right)^{-1} \bar{G}\left(\bar{U}^{(k)}, \Delta t\right) .
$$

Ideally, $M(\bar{U}, \Delta t)$ is both a good approximation of the system matrix, as well as a matrix for which an efficient (direct) solver is available.

\section{Optimal equivalent operator preconditioning}

Consider a linear advection-reaction-diffusion equation

$$
\partial_{t} U=\mathcal{A} U=D \partial_{x}^{2} U+\beta \partial_{x} U+\delta U,
$$

in which the diffusion, advection and reaction coefficients $D, \beta, \delta \in \mathbb{R}$ are taken constant over the domain. Without loss of generality, we consider $\beta>0$ throughout the text. In the equation-free setting of this paper, equation (3.1) is an unknown macroscopic equation, for which only a coarse time-stepper is available; however, for the analysis in the present section, we assume both the coarse and fine-scale model to be given by (3.1). We then define the time-stepper $s(U, \delta t)$, see equation (2.1), as a forward-Euler time-stepper for equation (3.1) with time-step $\delta t$; the corresponding coarse time-stepper is given by (2.6) in which $\mu=\mathcal{M}=I$.

As a preconditioner, we propose to use a different advection-reaction-diffusion equation

$$
\partial_{t} U=\mathcal{B} U=D^{*} \partial_{x}^{2} U+\beta^{*} \partial_{x} U+\delta^{*} U .
$$

Obviously, if we choose $D^{*}=D, \beta^{*}=\beta$ and $\delta^{*}=\delta$, we have $\mathcal{B}=\mathcal{A}$; however, when the coefficients of $\mathcal{A}$ are not known, this choice is not possible. Hence, we are led to investigate the effect of the parameters of the operator $\mathcal{B}$ on the convergence of iterative methods.

In [20], it was proposed to build a preconditioner using the leading part (the diffusion term), to which a linear reaction is added, i.e. (3.2) with $\beta^{*}=0$, in which $D^{*}$ is an estimate for the diffusion coefficient $D$, and $\delta^{*} \in \mathbb{R}$ is chosen to optimize the convergence rate. The paper [20] analyzed the case $D=D^{*}=1$. When $\delta=\delta^{*}=0$, the spectrum of $\mathcal{B}^{-1} \mathcal{A}$ then lies on the union of intervals $1 \pm i(0, \sqrt{2} \beta / 4 \pi]$; however, for some value $\delta^{*}=\delta^{-}=O\left(\beta^{2}\right)$ the spectrum of $\mathcal{B}^{-1} \mathcal{A}$ can be confined in a circle with radius $r^{-}<1 / 4$ and center $1-r^{-}$, independent of the value of $\beta$ [20]. Because the spectra of the preconditioned matrices are 
in a region that is bounded away from zero and independent from $\beta$ and $h$, the convergence rate of iterative methods is also independent of $\beta$ and the mesh size. For Richardson iteration, the convergence factor is minimized by choosing $\delta^{*}=\delta^{-}$. For Krylov methods, $\delta^{*}$ should typically be chosen much smaller [20]; however, an analytical treatment in that case is highly nontrivial, see e.g. [16].

A similar result was obtained for the space discretization $B_{h}^{-1} A_{h}$, both in the case of upwind and central discretizations on a mesh $\Pi=\left\{x_{0}=0, x_{1}=h, \ldots, x_{N-1}=(N-1) h, x_{N}=\right.$ $L\}$ with homogeneous Dirichlet boundary conditions; for the central discretizations, there is a requirement that that Peclet number $\gamma=\beta h /(2 D)<1$. In this paper, we only discuss centered finite differences in space; we refer to [20] for a comparison between an upwind and a centered discretization of the advection term.

We are interested in computing steady state solutions of equation (2.7). We discretize (3.1) and (3.2) in space and time to obtain a time-stepper that can be used in equation (2.9). To this end, we introduce the semi-discretized operators

$$
A_{h}=\operatorname{tridiag}\left(\frac{D}{h^{2}}-\frac{\beta}{2 h},-\frac{2 D}{h^{2}}+\delta, \frac{D}{h^{2}}+\frac{\beta}{2 h}\right),
$$

and

$$
B_{h}=\operatorname{tridiag}\left(\frac{D^{*}}{h^{2}}-\frac{\beta^{*}}{2 h},-\frac{2 D^{*}}{h^{2}}+\delta^{*}, \frac{D^{*}}{h^{2}}+\frac{\beta^{*}}{2 h}\right) .
$$

Clearly, the choice of the time discretization scheme will influence the spectral properties of the corresponding matrices. We give two examples. If we take the same discretization for the preconditioner, we are interested in the spectrum of

$$
\left(I_{h}-\left(I_{h}+B_{h} \delta t\right)^{k}\right)^{-1}\left(I_{h}-\left(I_{h}+A_{h} \delta t\right)^{k}\right) .
$$

Note that the preconditioning matrix will have an increased bandwidth. If we define a backward Euler scheme with time-step $\Delta t=k \delta t$ for the preconditioner, only linear systems with $B_{h}$ need to be solved [26]. The matrix to analyze is then given by

$$
\left(I_{h}-\left(I_{h}-B_{h} \Delta t\right)^{-1}\right)^{-1}\left(I_{h}-\left(I_{h}+A_{h} \delta t\right)^{k}\right) .
$$

\section{Spectral analysis for the continuous case}

In this section, we investigate the spectrum of the continuous operator $\mathcal{B}^{-1} \mathcal{A}$, which we consider on the domain $[0, L]$ with homogeneous Dirichlet boundary conditions. The eigenvectors of $\mathcal{A}$ are given by $u_{n}(x)=e^{-\beta / 2 D x} \sin (n \pi x / L)$, with corresponding real eigenvalues

$$
\lambda_{\mathcal{A}}^{n}=\delta-\frac{1}{4} \frac{\beta^{2}}{D}-D\left(\frac{n \pi}{L}\right)^{2} .
$$

Note that the operator $\mathcal{A}$ is a non-normal operator. The eigenstates $u_{n}(x)$ are not orthogonal. We denote the largest eigenvalue as $\lambda_{\mathcal{A}}^{>}=\delta-\beta^{2} / 4 D-D(\pi / L)^{2} ;$ if $\lambda_{\mathcal{A}}^{>}<0$, the solution decays in the long-term limit. 
In the following, we will denote the spectrum of an operator by $\Sigma(\cdot)$. We now proceed to characterize $\Sigma\left(\mathcal{B}^{-1} \mathcal{A}\right)$.

Lemma 4.1. Let $\mathcal{A}$ and $\mathcal{B}$ be defined as in (3.1), resp. (3.2). If $\mathcal{A}$ is stable, i.e. $\lambda_{\mathcal{A}}^{>}<0$, and $\delta^{*}$ is chosen such that $\delta^{-}<\delta^{*}<\delta^{+}$, with

$$
\delta^{ \pm}=\delta \frac{D^{*}}{D}-\beta^{2} \frac{D^{*}}{2 D^{2}}+\frac{\beta^{*} \beta}{2 D} \pm \frac{\left|D^{*} \beta-D \beta^{*}\right|}{D} \sqrt{-\lambda_{\mathcal{A}}^{>} / D}
$$

then all eigenvalues of the preconditioned system $\mathcal{B}^{-1} \mathcal{A}$ lie on a circle with radius

$$
r=\frac{\left(\beta-D \beta^{*} / D^{*}\right)^{2}}{4\left(D^{*} \delta-D \delta^{*}-\left(\beta^{*} / 2\right)\left(\beta-D \beta^{*} / D^{*}\right)\right)}
$$

that intersects the real axis at $D / D^{*}$.

Proof. As in [20], we rewrite the eigenvalue problem $\mathcal{B}^{-1} \mathcal{A} u=\mu u$ as

$$
(\mathcal{A}-\mu \mathcal{B}) u=0 .
$$

The values of $\mu$ for which the operator $\mathcal{A}-\mu \mathcal{B}$ has a zero eigenvalue correspond to eigenvalues of the preconditioned problem. By noting that the advection-diffusion-reaction operator

$$
\left(D-\mu D^{*}\right) \frac{d^{2}}{d x^{2}}+\left(\beta-\mu \beta^{*}\right) \frac{d}{d x}+\left(\delta-\mu \delta^{*}\right)
$$

is similar to $\mathcal{A}$ but with different constant coefficients, we obtain the condition

$$
\left(\delta-\mu \delta^{*}\right)-\frac{\left(\beta-\mu \beta^{*}\right)^{2}}{4\left(D-\mu D^{*}\right)}-\left(D-\mu D^{*}\right)\left(\frac{n \pi}{L}\right)^{2}=0,
$$

which we rewrite as a second-order polynomial in $\mu$,

$$
\left(\delta-\mu \delta^{*}\right)\left(D-\mu D^{*}\right)-\frac{\left(\beta-\mu \beta^{*}\right)^{2}}{4}-\left(D-\mu D^{*}\right)^{2}(n \pi / L)^{2}=0 .
$$

Before we analyze the solutions, we make a convenient transformation. Since the dominant term is $\left(D-\mu D^{*}\right)^{2}(n \pi / L)^{2}$ as $n \rightarrow \infty$, the zeros of (4.6) accumulate towards $D / D^{*}$. We therefore rewrite the equation with respect to this accumulation point and make the transformation

$$
\tilde{\mu}=\mu-\frac{D}{D^{*}}
$$

resulting in

$$
\left(D^{* 2}\left(\frac{n \pi}{L}\right)^{2}-\delta^{*} D^{*}+\frac{\beta^{* 2}}{4}\right) \tilde{\mu}^{2}+\left(D^{*} \delta-\delta^{*} D-\frac{\beta^{*}}{2}\left(\beta-\frac{D \beta^{*}}{D^{*}}\right)\right) \tilde{\mu}+\frac{1}{4}\left(\beta-\frac{D \beta^{*}}{D^{*}}\right)^{2}=0 .
$$

Around the accumulation point, the solutions are complex since the discriminant,

$$
\left(D^{*} \delta-\delta^{*} D-\frac{\beta^{*}}{2}\left(\beta-\frac{D \beta^{*}}{D^{*}}\right)\right)^{2}-\frac{1}{4}\left(\beta-\frac{D \beta^{*}}{D^{*}}\right)^{2}\left(D^{* 2}\left(\frac{n \pi}{L}\right)^{2}-\beta^{*} D^{*}+\frac{\beta^{* 2}}{4}\right)
$$


is negative for large $n$.

All the eigenvalues of the preconditioned system fit the polynomial equation (4.7). For a given $n$, the equation has a negative discriminant (4.8) if $\delta^{*}$ is chosen between

$$
\delta_{n}^{ \pm}=\delta D^{*} / D-\beta^{2} D^{*} /\left(2 D^{2}\right)+\frac{\beta^{*} \beta}{2 D} \pm \frac{\left|D^{*} \beta-D \beta^{*}\right|}{D} \sqrt{-\frac{\delta}{D}+\frac{\beta^{2}}{4 D^{2}}+\left(\frac{n \pi}{L}\right)^{2}} .
$$

The term under the square root is recognized as $-\lambda_{\mathcal{A}}^{n} / D$, with $\lambda_{\mathcal{A}}^{n}<0$ an eigenvalue of $\mathcal{A}$. The square root is therefore real and, for all $n$, both $\delta_{n}^{+}$and $\delta_{n}^{-}$are real.

It is also clear that $\delta_{n}^{-}<\delta_{n-1}^{-}<\ldots<\delta_{1}^{-}$and $\delta_{1}^{+}<\delta_{2}^{+}<\ldots<\delta_{n}^{+}$.

If $\delta^{*}$ is chosen such that $\delta_{1}^{-}<\delta^{*}<\delta_{1}^{+}$, then the discriminant is negative for each $n$ and all the solutions of equation (4.7) are complex conjugate and satisfy, with the help of Vieta's root formula,

$$
\tilde{\mu}_{n}+\tilde{\mu}_{n}^{*}=-\frac{D^{*} \delta-D \delta^{*}-\left(\beta^{*} / 2\right)\left(\beta-D \beta^{*} / D^{*}\right)}{D^{* 2}(n \pi / L)^{2}-D^{*} \delta^{*}+\beta^{* 2} / 4}
$$

and

$$
\tilde{\mu}_{n} \tilde{\mu}_{n}^{*}=\frac{\left(\beta-D \beta^{*} / D^{*}\right)^{2}}{4\left(D^{* 2}(n \pi / L)^{2}-D^{*} \delta^{*}+\beta^{*^{2}} / 4\right)} .
$$

We now proceed to prove that the eigenvalues $\tilde{\mu}_{n}$ lie on a circle that touches the origin. When we assume the circle to be in $\mathbb{C}^{-}$, we write $\tilde{\mu}_{n}=r\left(e^{i \phi}-1\right)$ we find a radius

$$
r=\frac{\left(\beta-D \beta^{*} / D^{*}\right)^{2}}{4\left(D^{*} \delta-D \beta^{*}-\left(\beta^{*} / 2\right)\left(\beta-D \beta^{*} / D^{*}\right)\right)},
$$

independently of $n$; hence the spectrum lies on a circle. If $r<0$, the spectrum lies on a circle in $\mathbb{C}^{+}$. We then conclude the proof by performing the substitution $\mu=\tilde{\mu}+d$.

The case $\beta^{*}=0$. When we set $\beta^{*}=0$, we obtain the optimal equivalent preconditioner that was proposed by Manteuffel and Otto [20]. The choice $\delta^{*}=\delta^{-}$is then the optimal choice proposed by Manteuffel and Otto in [20] for the Richardson iteration. It corresponds to the minimal radius of circles

$$
r^{-}=\frac{\beta^{2}}{4\left(\delta D^{*}-\delta^{-} D\right)}=\frac{D}{4 D^{*}} \frac{2}{1+\sqrt{1-4 \delta D / \beta^{2}+4 \pi^{2} D^{2} /\left(\beta^{2} L^{2}\right)}} .
$$

Example 4.2. We now illustrate the dependence of the spectrum of $\mathcal{B}^{-1} \mathcal{A}$ on $\delta^{*}$. Consider operator $\mathcal{A}$, with $D=1, \beta=3$ and $\delta=0.3$ on a domain of length $L=5$, preconditioned with an operator $\mathcal{B}$ with $D^{*}=0.5$ and $\beta^{*}=0$. We find that a $\delta^{*} \in\left[\delta^{-}=-4.396, \delta^{+}=0.1969\right]$ leads to problems where the eigenvalues lie on a circle. We compute the eigenvalues $\mu_{n}$ up to $n=100$ via equations (4.10) and (4.11) for $\delta^{*} \in\left\{\delta^{-},-3,-1, \delta^{+}\right\}$, see figure 1 (left). For all choices of $\delta^{*}$, the circle intersects the real axis at $D / D^{*}=2$. For $\delta^{*}=\delta^{-}$, the circle lies to the left of this point, for $\delta^{*}=\delta^{+}$it lies to the right. For $\delta^{*}=\delta^{-}$, the circle has a radius $r^{-}=0.49$ and for $\delta^{*}=\delta^{+}$, the radius is $r^{+}=-47.8$. (We keep the sign in equation (4.3) 

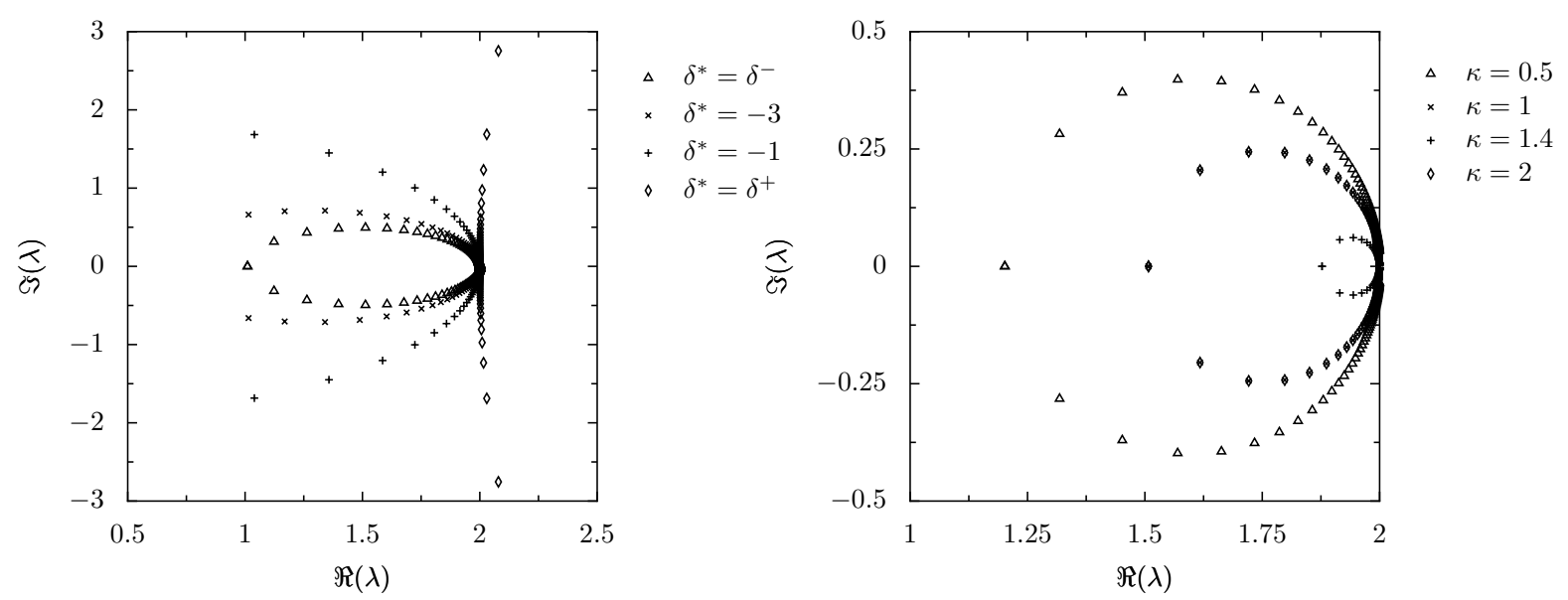

Figure 1: The eigenvalues of the preconditioned system $\mathcal{B}^{-1} \mathcal{A}$ for example 4.2 (left) and example 4.3 (right). All the eigenvalues lie on circles with different radii.

to indicate circles that lie to the right of the accumulation point.) For $\delta^{*}=-1$, the radius of the circle is approximately equal to 2, and it almost touches the origin. The eigenvalues, however, do not lie on the complete curve. Note that for some choice of $\delta^{*}$ the radius will become infinite. For $\delta^{*} \notin\left[\delta^{-}, \delta^{+}\right]$, real eigenvalues appear at the leftmost part of the circles.

When $\beta \rightarrow \infty$, we can simplify the equation for $\delta^{ \pm}$in (4.9) to

$$
\delta^{ \pm}=\delta \frac{D^{*}}{D}-\beta^{2} \frac{D^{*}}{2 D^{2}} \pm \beta^{2} \frac{D^{*}}{2 D^{2}} .
$$

For $\delta^{*}=\delta^{-}=\delta D^{*} / D-\beta^{2} D^{*} / D^{2}$, we then have $r^{-}=D /\left(4 D^{*}\right)$, and for $\delta^{*} \rightarrow \delta^{+}=\delta D^{*} / D$ the radius $r^{+} \rightarrow \infty$. In this case, when $\delta=\delta^{-}$, the eigenvalues lie on a circle with a radius $D /\left(4 D^{*}\right)$ and center $3 D /\left(4 D^{*}\right)$, and the associated Richardson convergence factor $\rho^{-}=r^{-} /\left(d-r^{-}\right)=1 / 3$, with $d=D / D^{*}$. We observe that, in this limit, the convergence of Richardson iteration is unaffected by choosing $D^{*} \neq D$. For the equation-free setting, where $D, \beta$ and $\delta$ are not known explicitly, one can therefore, in this continuous case, simply fix $D^{*}$ and one only needs to tune $\delta^{*}$ to optimize the convergence factor for the given problem.

The case $\beta^{*}>0$. When $\beta^{*}>0$, the preconditioner becomes a non-symmetric operator, requiring more computational effort to solve. In the equation-free setting, however, this is not problematic, since the cost of the computation of a matrix-vector product using the coarse time-stepper can be huge. We therefore really want to minimize the number of preconditioned iterations, and we can safely neglect the work that is done in solving linear systems with the preconditioner. 
For $\beta^{*} \rightarrow \beta / d$, we see that $\delta^{ \pm} \rightarrow \delta / d$, and correspondingly $r^{ \pm} \rightarrow 0$ and $\rho^{ \pm} \rightarrow 0$. In this limit, $\mathcal{B}=\mathcal{A} / d$, and $\mathcal{B}^{-1} \mathcal{A}=\mathcal{I} / d$; hence preconditioning is perfect.

We illustrate the dependence of the spectrum of $\mathcal{B}^{-1} \mathcal{A}$ on $\beta^{*}$.

Example 4.3. Consider operator $\mathcal{A}$, with $D=1, \beta=3$. and $\delta=0.3$ on a domain of length $L=5$, preconditioned with an operator $\mathcal{B}$ with $D^{*}=0.5$. We again compute the eigenvalues $\mu_{n}$ up to $n=100$ via equations (4.10) and (4.11) for $\beta^{*} \in\{0,1,1.4,2\}$, see figure 1 (right), each time using $\delta^{*}=\delta^{-}\left(\beta^{*}\right)$. Again, for all choices of $\beta^{*}$, the circle intersects the real axis at $D / D^{*}=2$. For $\beta^{*} \rightarrow \beta / d$, the radius of the circles $r^{-} \rightarrow 0$. Note that the spectra for $\beta^{*}=1$ and $\beta^{*}=2$ are identical, due symmetry around $\beta^{*}=1.5=\beta / d$, the value for which $\mathcal{B}^{-1} \mathcal{A}=\mathcal{I} / d$.

As a final remark, we note that one should expect to see superlinear convergence for Krylov methods [1], since most of the eigenvalues will lie on the right hand side of the circle and will accumulate near $d$.

\section{Spectral analysis for the discrete case}

\subsection{A single forward Euler time step}

We now turn to the discrete case (3.5), in which we first consider $k=1(\Delta t=\delta t)$ for simplicity; we introduce the discretization parameter $h=N / L$ and the Peclet number $\gamma=\beta h /(2 D), \gamma^{*}=\beta^{*} h /\left(2 D^{*}\right), \tilde{\delta}=\delta / D$, and $\tilde{\delta}^{*}=\delta^{*} / D^{*}$. We then have

$$
\begin{aligned}
& \tilde{A}_{h}=h^{2} A_{h}=D \cdot \operatorname{tridiag}\left(1-\gamma,-2+\tilde{\delta} h^{2}, 1+\gamma\right), \\
& \tilde{B}_{h}=h^{2} B_{h}=D^{*} \cdot \operatorname{tridiag}\left(1-\gamma^{*},-2+\tilde{\delta}^{*} h^{2}, 1+\gamma^{*}\right),
\end{aligned}
$$

and the matrix (3.5) then reduces to $\tilde{B}_{h}^{-1} \tilde{A}_{h} \in \mathbb{R}^{N \times N}$. We provide some results for more general cases in the subsequent sections.

The eigenvalues of the preconditioned matrix $\tilde{B}_{h}^{-1} \tilde{A}_{h}$ are $\mu \in \mathbb{C}$ for which $\tilde{A}_{h}-\mu \tilde{B}_{h}$ has an eigenvalue zero. In the following, we will denote the spectrum of a matrix by $\Sigma(\cdot)$. As for the continuous case, we now proceed to characterize $\Sigma\left(\tilde{B}_{h}^{-1} \tilde{A}_{h}\right)$.

Lemma 5.1. Let $\tilde{A}_{h}$ and $\tilde{B}_{h}$ be tridiagonal $N \times N$ matrices as defined in (5.1), resp. (5.2). If $\Sigma\left(A_{h}\right) \subset \mathbb{C}^{-}, \gamma<1$, and $\delta^{-}<\delta^{*}<\delta^{+}$, with $\delta^{-}, \delta^{+} \in \mathbb{R}$ defined below and $d=D / D^{*}$, then all the eigenvalues of the preconditioned system $\tilde{B}_{h}^{-1} \tilde{A}_{h}$ lie on a circle with radius

$$
r=\frac{1}{2 D^{*}} \frac{\left(\delta^{*} D-\delta D^{*}\right)^{2} h^{4}-\left[\left(2 D^{*}-\delta^{*} h^{2}\right) D \gamma-\left(2 D-\delta h^{2}\right) D^{*} \gamma^{*}\right]^{2}}{\left(2 \delta^{*}\right)\left(\left(\delta^{*} D-\delta D^{*}\right) h^{2}+\gamma^{*}\left[\left(2 D^{*}-\delta^{*} h^{2}\right) D \gamma-\left(2 D-\delta h^{2}\right) D^{*} \gamma^{*}\right]\right)}
$$

that intersects the real axis at

$$
\mu_{*}=\frac{2 D-\delta h^{2}}{2 D^{*}-\delta^{*} h^{2}}
$$


Proof. The eigenvalues of a tridiagonal $N$ by $N$ matrix $\mathrm{M}=\operatorname{tridiag}(b, a, c)$ are given by

$$
\lambda_{M}^{n}=a-2 \sqrt{b c} \cos (n \pi /(N+1))
$$

with $n=1,2, \ldots, N$. Applied to the matrix $A_{h}$, as defined in (3.3), the eigenvalues are

$$
\lambda_{A_{h}}^{n}=-2 D / h^{2}+\delta-2 \sqrt{D^{2} / h^{4}-\beta^{2} / 4 h^{2}} \cos (n \pi /(N+1)) .
$$

Depending on the sign of $4 D^{2}-\beta^{2} h^{2}$, these eigenvalues either lie on the negative real axis or on a line parallel with the imaginary axis intersecting the real axis in $-2 D / h^{2}+\delta$. We look for the values of $\mu$ that satisfy

$\tilde{A}_{h}-\mu \tilde{B}_{h}=D \cdot \operatorname{tridiag}\left(1-\gamma,-2+\tilde{\delta} h^{2}, 1+\gamma\right)-\mu D^{*} \cdot \operatorname{tridiag}\left(1-\gamma^{*},-2+\tilde{\delta}^{*} h^{2}, 1+\gamma^{*}\right)=0$.

We introduce $\hat{\mu}=\mu D^{*} / D$, and look for $\hat{\mu}$ as the solutions of

$$
-2(1-\hat{\mu})+h^{2}\left(\tilde{\delta}-\hat{\mu} \tilde{\delta}^{*}\right)-2 t_{n} \sqrt{(1-\hat{\mu})^{2}-\left(\gamma-\hat{\mu} \gamma^{*}\right)^{2}}=0, \quad n=1, \ldots, N
$$

with $n=1, \ldots, N$ and $t_{n}=\cos \left(\frac{n \pi}{N+1}\right)$. Since $\cos (n \pi /(N+1))=-\cos ((N+1-n) \pi /(N+1))$, this is equivalent to

$$
\begin{aligned}
& \left(-2(1-\hat{\mu})+h^{2}\left(\tilde{\delta}-\hat{\mu} \tilde{\delta}^{*}\right)-2 t_{n} \sqrt{(1-\hat{\mu})^{2}-\left(\gamma-\hat{\mu} \gamma^{*}\right)^{2}}\right) \\
& \times\left(-2(1-\hat{\mu})+h^{2}\left(\tilde{\delta}-\hat{\mu} \tilde{\delta}^{*}\right)+2 t_{n} \sqrt{(1-\hat{\mu})^{2}-\left(\gamma-\hat{\mu} \gamma^{*}\right)^{2}}\right)=0 .
\end{aligned}
$$

So, $\Sigma\left(\tilde{B}_{h}^{-1} \tilde{A}_{h}\right)$ is formed by the solutions of

$$
-\left(-2(1-\hat{\mu})+h^{2}\left(\tilde{\delta}-\hat{\mu} \tilde{\delta}^{*}\right)\right)^{2}+4\left((1-\hat{\mu})^{2}-\left(\gamma-\hat{\mu} \gamma^{*}\right)^{2}\right) t_{n}^{2}=0,
$$

with $n \leq N / 2$.

The analysis of the solutions of equation (5.6) is easier if we first make a transformation of variables from $\hat{\mu}$ to $\hat{\nu}$,

$$
\hat{\mu}=\hat{\nu}+\frac{2-\tilde{\delta} h^{2}}{2-\tilde{\delta}^{*} h^{2}}
$$

where the shift is found by solving equation (5.5) for $\hat{\mu}$ with $t_{n}=0$. This transformation leads to a second order polynomial in the new variable $\hat{\nu}$,

$$
a_{n} \hat{\nu}_{n}^{2}+b_{n} \hat{\nu}_{n}+c_{n}=0
$$

with coefficients

$$
\begin{aligned}
& a_{n}=\left(2-\tilde{\delta}^{*} h^{2}\right)^{2}-4 t_{n}^{2}\left(1-\gamma^{* 2}\right), \\
& b_{n}=\frac{-8 t_{n}^{2}}{2-\tilde{\delta}^{*} h^{2}}\left(\left(\tilde{\delta}^{*}-\tilde{\delta}\right) h^{2}+\gamma^{*}\left[\left(2-\tilde{\delta}^{*} h^{2}\right) \gamma-\left(2-\tilde{\delta} h^{2}\right) \gamma^{*}\right]\right), \\
& c_{n}=\frac{4 t_{n}^{2}}{\left(2-\tilde{\delta}^{*} h^{2}\right)^{2}}\left(\left[\left(2-\tilde{\delta}^{*} h^{2}\right) \gamma-\left(2-\tilde{\delta} h^{2}\right) \gamma^{*}\right]^{2}-\left(\tilde{\delta}^{*}-\tilde{\delta}\right)^{2} h^{4}\right) .
\end{aligned}
$$


Again the application of Vieta's root formula and writing $\hat{\nu}_{n}=\hat{r}\left(e^{i \phi}-1\right)$ we find a radius

$$
\hat{r}=c_{n} / b_{n}=\frac{1}{2} \frac{\left(\tilde{\delta}^{*}-\tilde{\delta}\right)^{2} h^{4}-\left[\left(2-\tilde{\delta}^{*} h^{2}\right) \gamma-\left(2-\tilde{\delta} h^{2}\right) \gamma^{*}\right]^{2}}{\left(2-\tilde{\delta}^{*} h^{2}\right)\left((\tilde{\delta} * \tilde{\delta}) h^{2}+\gamma^{*}\left[\left(2-\tilde{\delta}^{*} h^{2}\right) \gamma-\left(2-\tilde{\delta} h^{2}\right) \gamma^{*}\right]\right)} .
$$

This radius is independent of $n$, which suggests that the solutions lie on circle. This, however, is only true if all eigenvalues form complex conjugate pairs. To check this, we consider the discriminant of the equation (5.8), which is given as

$$
\begin{aligned}
\mathcal{D}_{n}= & b_{n}^{2}-4 a_{n} c_{n} \\
= & 16 t_{n}^{2}\left(h^{4}\left(1-\gamma^{2}\right)\left(\tilde{\delta}^{*}\right)^{2}+2 h^{2}\left(2 \gamma^{2}-\tilde{\delta} h^{2}-\left(2-\tilde{\delta} h^{2}\right) \gamma \gamma^{*}\right) \tilde{\delta}^{*}\right. \\
& \left.+4\left(\gamma-\gamma^{*}\right)^{2}\left(\left[2 \gamma-\gamma^{*}\left(2-\tilde{\delta} h^{2}\right)\right]^{2}-\left(\gamma^{*} \tilde{\delta} h^{2}\right)^{2}\right)\right) .
\end{aligned}
$$

Depending on the choice of $\tilde{\delta}^{*}, \mathcal{D}_{n}$ changes sign. Only if $\mathcal{D}_{n} \leq 0$, the solutions lie on the circle. Since $\mathcal{D}_{n}$ is a quadratic function of $\delta^{*}$, its value is negative between the roots of $\mathcal{D}_{n}=0$, provided the term leading $\delta^{* 2}$ is positive. This is the case for $\gamma<1$.

The interval of possible choices of $\tilde{\delta}^{*}$ that make $\mathcal{D}_{n}<0$ for all $n \leq N / 2$ is determined by $n=1$. Setting $\mathcal{D}_{1}=0$ with $n=1$ and solving for $\tilde{\delta}^{*}$ leads to two solutions

$$
\tilde{\delta}^{ \pm}=\frac{-\left(2 \gamma^{2}-\tilde{\delta} h^{2}-\left(2-\tilde{\delta} h^{2}\right) \gamma \gamma^{*}\right) \pm\left|\gamma-\gamma^{*}\right| \sqrt{\left(2-\tilde{\delta} h^{2}\right)^{2}-4\left(1-\gamma^{2}\right) t_{1}^{2}}}{h^{2}\left(1-\gamma^{2}\right)} .
$$

These bounds on $\tilde{\delta}^{*}$ are real if the term under the square root (which is proportional to the product $\left.\lambda_{A_{h}}^{1} \cdot \lambda_{A_{h}}^{N}\right)$ is positive. Since we have assumed $\Sigma\left(A_{h}\right) \in \mathbb{C}^{-}$, this is always the case.

When scaling back from $\hat{\mu}$ to $\mu$, we find that the eigenvalues $\mu$ lie on a circle with radius

$$
r=\frac{D}{D^{*}} \hat{r}=\frac{D}{2 D^{*}} \frac{\left(\tilde{\delta}^{*}-\tilde{\delta}\right)^{2} h^{4}-\left[\left(2-\tilde{\delta}^{*} h^{2}\right) \gamma-\left(2-\tilde{\delta} h^{2}\right) \gamma^{*}\right]^{2}}{\left(2-\tilde{\delta}^{*} h^{2}\right)\left(\left(\tilde{\delta}^{*}-\tilde{\delta}\right) h^{2}+\gamma^{*}\left[\left(2-\tilde{\delta}^{*} h^{2}\right) \gamma-\left(2-\tilde{\delta} h^{2}\right) \gamma^{*}\right]\right)}
$$

that intersects with the real axis at

$$
\mu=\mu_{*}=\frac{2 D-\delta h^{2}}{2 D^{*}-\delta^{*} h^{2}} .
$$

To conclude the proof, we now rewrite these formulae in terms of the original $\delta, \delta^{*}, D$ and $D^{*}$, and introduce $d=D / D^{*}$, to obtain

$$
\begin{aligned}
r & =\frac{1}{2 D^{*}} \frac{\left(\delta^{*} D-\delta D^{*}\right)^{2} h^{4}-\left[\left(2 D^{*}-\delta^{*} h^{2}\right) D \gamma-\left(2 D-\delta h^{2}\right) D^{*} \gamma^{*}\right]^{2}}{\left(2 D^{*}-\delta^{*} h^{2}\right)\left(\left(\delta^{*} D-\delta D^{*}\right) h^{2}+\gamma^{*}\left[\left(2 D^{*}-\delta^{*} h^{2}\right) D \gamma-\left(2 D-\delta h^{2}\right) D^{*} \gamma^{*}\right]\right)} \\
& =\frac{1}{2} \frac{\left(\delta^{*} d-\delta\right)^{2} h^{4}-\left[\left(2 D^{*}-\delta^{*} h^{2}\right) d \gamma-\left(2 D-\delta h^{2}\right) \gamma^{*}\right]}{\left(2 D^{*}-\delta^{*} h^{2}\right)\left(\left(\delta^{*} d-\delta\right) h^{2}+\gamma^{*}\left[\left(2 D^{*}-\delta^{*} h^{2}\right) d \gamma-\left(2 D-\delta h^{2}\right) \gamma^{*}\right]\right)} .
\end{aligned}
$$




\begin{tabular}{c|c|c|c|c}
\hline$d$ & $D^{*}$ & $\delta^{-}$ & $r^{-}$ & $\rho^{-}$ \\
\hline 0.5 & 2.0 & -116.671 & 0.0867 & 0.2095 \\
1.0 & 1.0 & -58.335 & 0.1734 & 0.2095 \\
2.0 & 0.5 & -29.168 & 0.3468 & 0.2095 \\
\hline
\end{tabular}

Table 1: Optimal choice of $\delta^{*}$ for example 5.2, radius of corresponding spectrum of $B_{h}^{-1} A_{h}$, and associated Richardson convergence factor, as a function of $d=D / D^{*}$.

and

$$
\delta^{ \pm}=\frac{1}{d} \frac{-\left(2 D \gamma^{2}-\delta h^{2}-\left(2 D-\delta h^{2}\right) \gamma \gamma^{*}\right) \pm\left|\gamma-\gamma^{*}\right| \sqrt{\left(-2 D+\delta h^{2}\right)^{2}-4 D^{2}\left(1-\gamma^{2}\right) t_{1}^{2}}}{h^{2}\left(1-\gamma^{2}\right)} .
$$

If the limit $h \rightarrow 0$ is taken with all other parameters fixed, the point where the circle intersects with the real axis, $\mu_{*}=\left(-2 D+h^{2} \delta\right) /\left(-2 D^{*}+h^{2} \delta^{*}\right)$, shifts to $d=D / D^{*}$, our result for the continuous case. Similarly, it can also be checked that the radius of the circle approaches our continuous result. Hence, for sufficiently small $h$, one can bound the radius of the circles - and the associated convergence factor - independently of $\beta$ and $h$.

\subsection{Numerical illustration}

We now proceed to illustrate this result numerically. We first show that the resulting preconditioners are unaffected by a scaling of all coefficients.

Example 5.2. Consider the matrix $A_{h}$ using $D=1, \beta=10, \delta=5, h=2 \cdot 10^{-2}$, and $N=49$, leading to $\gamma=0.1$. We define the preconditioner $B_{h}$ using $D^{*}=D / d$ and $\beta^{*}=4 / d$, with $d=0.5,1,1.5$, and compute the corresponding value of $\delta^{-}$and $r^{-}$, as well as the associated Richardson convergence factor $\rho^{-}$. The results are given in table 1 . It is clear that the optimal choice $\delta^{*}=\delta^{-}$is simply scaled with $1 / d$ (and hence the resulting optimal preconditioner is identical up to scaling of all values with $d$ ). The radius is then scaled with $d$ and the Richardson convergence factor is independent of $d$.

Next, we look at the effect of using $\gamma^{*} \neq \gamma$ on the optimal parameters and associated Richardson convergence rate.

Example 5.3. Consider the matrix $A_{h}$ using $D=1, \beta=2 \gamma D / h, \delta=5, h=2 \cdot 10^{-2}$, and $N=49$ with a Peclet number $\gamma \in\{0.05,0.1,0.15,0.2\}$. We define the preconditioner $B_{h}$ using $D^{*}=1$ and $\beta^{*} \in[-10,30]$, i.e. $\gamma^{*} \in[-0.1,0.3]$, and compute the corresponding value 

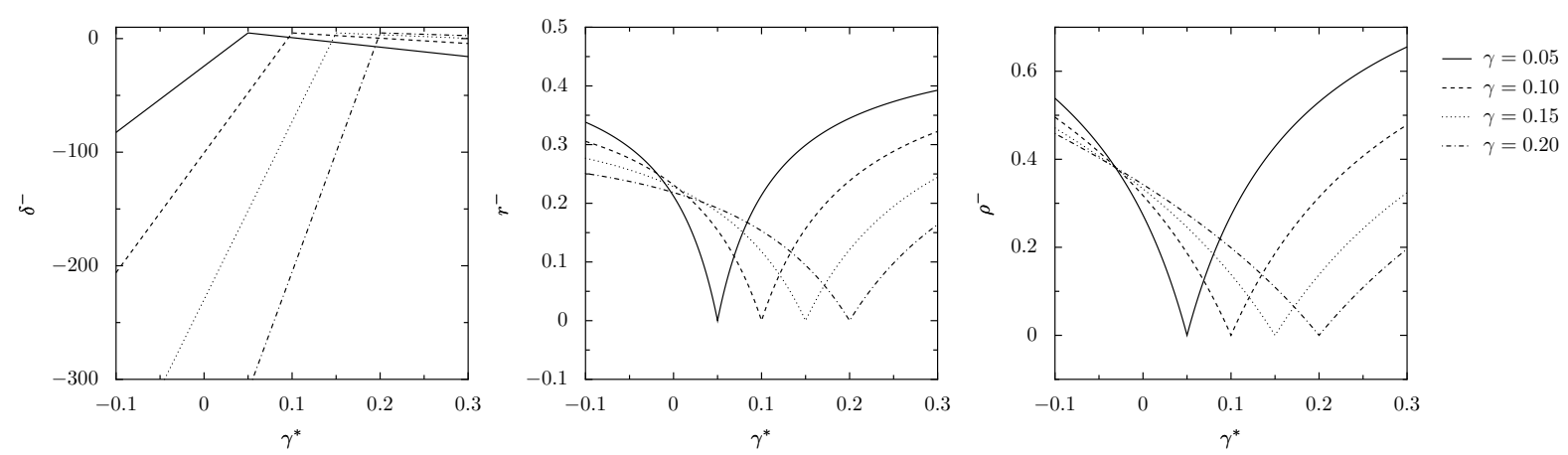

Figure 2: Left: Optimal choice of $\delta^{*}$ for example 5.3 as a function of $\beta^{*}$. Middle: Corresponding radius of the spectrum of $B_{h}^{-1} A_{h}$. Right: Associated Richardson convergence factor.

of $\delta^{-}$and $r^{-}$, as well as the Richardson convergence factor $\rho^{-}$. The results are shown in figure 2. The figures show that, when $\gamma^{*}<\gamma, \delta^{-}$rapidly attains large negative values as a function of $\gamma$ (for fixed values of $\gamma^{*}$ ), whereas for $\gamma^{*}>\gamma$, the absolute value of $\delta^{-}$increases much more slowly. In both cases, $\delta^{-}$depends linearly on $\left|\gamma-\gamma^{*}\right|$ (for fixed $\gamma$ ). Clearly, the radius of the circles $r^{-}$, and the corresponding optimal convergence rate $\rho^{-}$, decrease as $\left|\gamma-\gamma^{*}\right|$ decreases. For $\gamma^{*}=0$, the values of $\delta^{-}$are in agreement with the results of Manteuffel in [20]. We also recover the result of [20] that, for $\gamma^{*}=0, r^{-}<1 / 4$, and $\rho^{-}<1 / 3$.

Next, we illustrate the effects of deviating from optimality on the convergence factors.

Example 5.4. Consider the matrix $A_{h}$ using $D=1, \beta=15, \delta=5, h=2 \cdot 10^{-2}$, and $N=$ 49, leading to $\gamma=0.15$. We define the preconditioner $B_{h}$ using $D^{*}=1$ and $\beta^{*}=2 \gamma^{*} D / h$, using $\gamma^{*}=0,0.1,0.2,0.3$, and $\delta^{*} \in\left[\delta^{-}\left(\gamma^{*}\right), \delta^{+}\left(\gamma^{*}\right)\right]$, and compute the corresponding value of $r$ and $\rho$ as a function of $\delta^{*}$. The results are shown in figure 3, where, for each $\gamma^{*}$, the curves are only drawn in the interval $\left[\delta^{-}\left(\gamma^{*}\right), \delta^{+}\left(\gamma^{*}\right)\right]$. The vertical asymptote is due to the change of orientation of the circles within the interval $\left[\delta^{-}\left(\gamma^{*}\right), \delta^{+}\left(\gamma^{*}\right)\right]$. The figures show that, although the optimal convergence factor is smaller when $\gamma^{*}$ is closer to $\gamma$, the range of possible $\delta^{*}$ for which the spectrum is determined by the circles derived above, is smaller (and eventually collapses to a single point $\left(\delta^{*}=\delta\right)$ when $\left.\gamma^{*}=\gamma\right)$. Also, the range of $\delta^{*}$ for which $\rho$ is reasonably insensitive to changes in $\delta^{*}$ is significantly smaller when $\gamma^{*} \rightarrow \gamma$. Thus, when the parameters of $A_{h}$ are not exactly known, choosing $\gamma^{*}$ not too close to $\gamma$ might yield more robustness. However, note that the real eigenvalues that appear when $\delta^{*}<\delta^{-}$do not necessarily impact convergence dramatically as will be illustrated in section 6.

Given the freedom in all preconditioning parameters, and the difficulty in selecting a convenient $\delta^{*}$ once $D^{*}$ and $\gamma^{*}$ have been chosen, one could be inclined to simply put $\delta^{*}=0$, and look at the convergence factor as a function of $\gamma^{*}$. The next example investigates this 

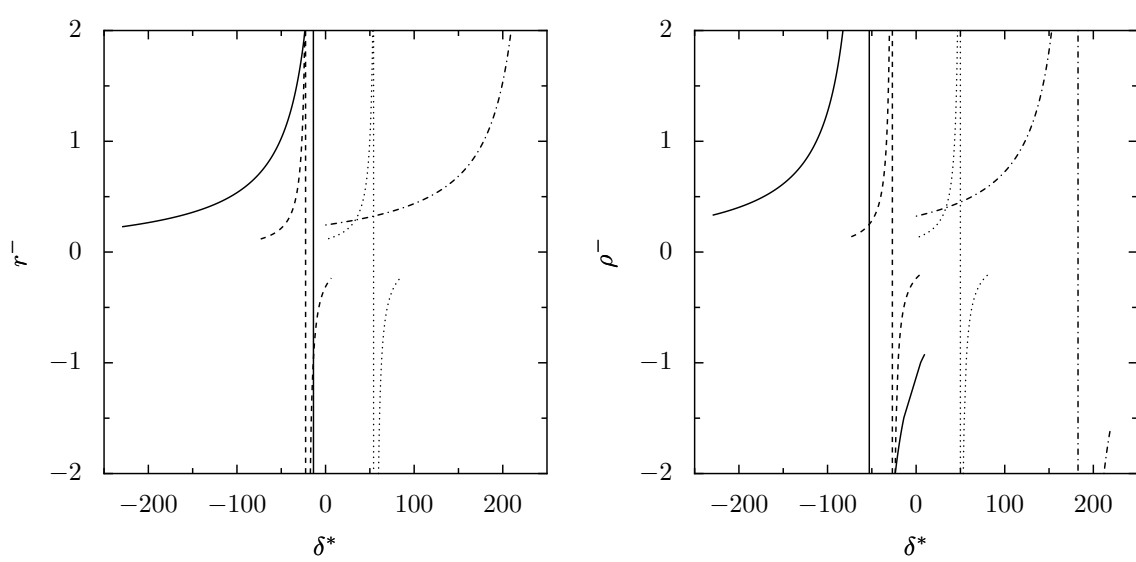

$$
\begin{aligned}
-\gamma^{*} & =0.00 \\
\cdots-\gamma^{*} & =0.10 \\
\cdots \cdots & \gamma^{*}=0.20 \\
\cdots \cdots \gamma^{*} & =0.30
\end{aligned}
$$

Figure 3: Left: Radius of the spectrum of $B_{h}^{-1} A_{h}$ for example 5.4 as a function of $\delta^{*}$. Right: Associated Richardson convergence factor.
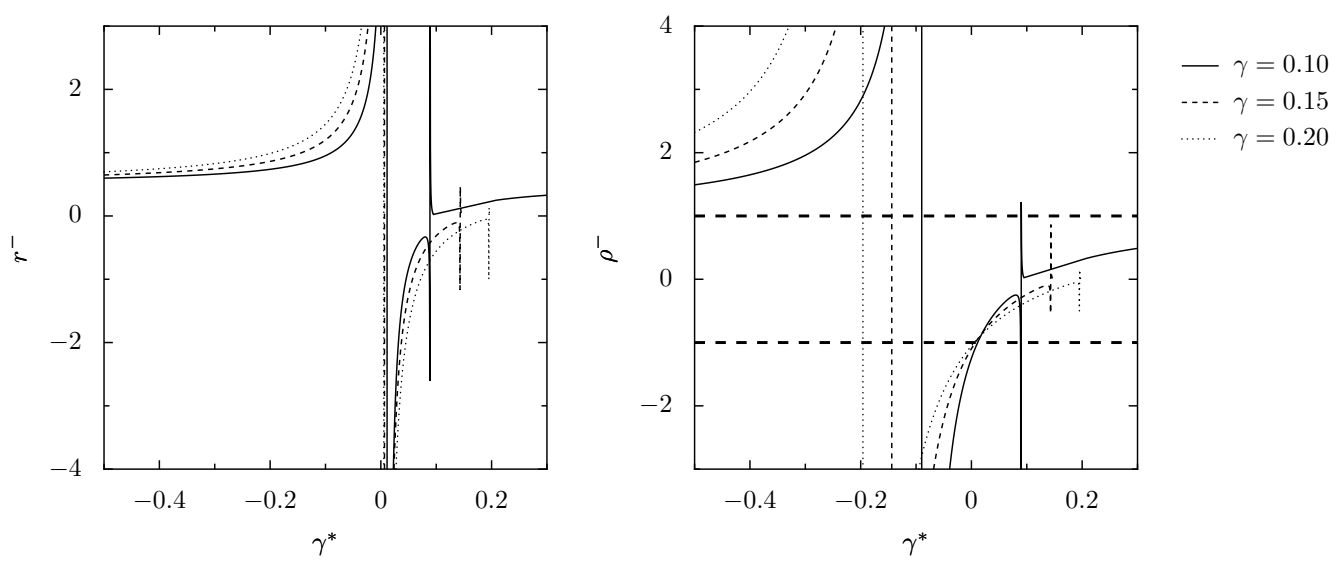

Figure 4: Left: Radius of the spectrum of $B_{h}^{-1} A_{h}$ for example 5.5 as a function of $\delta^{*}$. Right: Associated Richardson convergence factor. The thick dashed lines indicate the values $\rho= \pm 1$.

choice.

Example 5.5. Consider the matrix $A_{h}$ using $D=1, \beta \in\{10,15,20\}, \delta=5, h=2 \cdot 10^{-2}$, and $N=49$. We define the preconditioner $B_{h}$ using $D^{*}=1$ and $\delta^{*}=0 \beta^{*}=2 \gamma^{*} D / h$, using $\gamma^{*} \in[-0.5,0.3]$ and compute the corresponding values of $r$ and $\rho$ as a function of $\delta^{*}$. The results are shown in figure 4 , where, for each $\gamma$, the curves are only drawn for the values of $\gamma^{*}$ for which $\delta^{*}=0 \in\left[\delta^{-}\left(\gamma^{*}\right), \delta^{+}\left(\gamma^{*}\right)\right]$. The vertical asymptotes are due to changes of orientation of the circles within the interval $\left[\delta^{-}\left(\gamma^{*}\right), \delta^{+}\left(\gamma^{*}\right)\right]$. We see that, depending on $\beta$, there is a range of values of $\gamma^{*}$ for which the choice $\delta^{*}=0$ can lead to a reasonable convergence factor. 

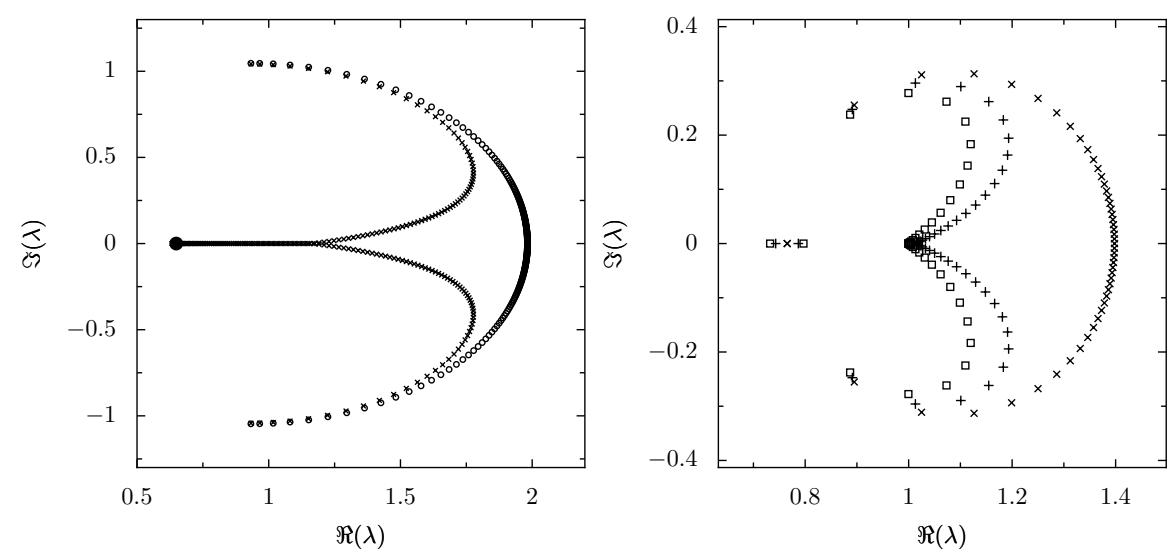

$\times \quad k=1$

$+\quad k=5$

口 $\quad k=10$

Figure 5: Left: The eigenvalues of the system using a forward Euler preconditioner using $k=1$ (crosses) and $k=2$ (circles) steps. The value of $\mu^{*}$ for $k=2$ is indicated with a black dot. Right: Spectrum of (3.5) for increasing $k$ with $\gamma=0.2$ and $\nu=0.2$.

\subsection{Multiple time steps preconditioned with forward Euler}

If we consider two forward Euler steps $(k=2)$, the situation immediately becomes much more complicated. Starting from (3.5) and substituting $\nu=\delta t / h^{2}$, we have

$$
\left(I_{h}-\left(I_{h}+B_{h} \delta t\right)^{2}\right)^{-1}\left(I_{h}-\left(I_{h}+A_{h} \delta t\right)^{2}\right)=\left(\tilde{B}_{h}+\frac{\nu}{2} \tilde{B}_{h}^{2}\right)^{-1}\left(\tilde{A}_{h}+\frac{\nu}{2} \tilde{A}_{h}^{2}\right),
$$

which can be seen as the discretization of a fourth order equation that depends on $\nu$. Finding the eigenvalues $\mu$ of (5.20) is equivalent to finding the values of $\mu$ for which

$$
\left(\tilde{A}_{h}-\mu \tilde{B}_{h}\right)+\frac{\nu}{2}\left(\tilde{A}_{h}^{2}-\mu \tilde{B}_{h}^{2}\right)=0 .
$$

For $k>1$, we will only obtain approximations to some particular points in the spectrum.

A comparison of the spectrum for $k=1$ and $k=2$ for a problem with $L=15, D=1$, $D^{*}=0.5, \beta=3, \delta^{*}=-2, \delta=0.3$ and $\delta t=0.0017$ and $N=231(\nu=0.403)$ is give in figure 5 (left). We see that for the leftmost eigenvalues, there is agreement between $k=1$ and $k=2$. This can be understood by looking at behavior for smooth modes. For modes $u$ where $u^{T} A_{h} u \gg u^{T} A_{h}^{2} u$ and $u^{T} B_{h} u \gg u^{T} B_{h}^{2} u$ we can make the following approximation

$$
\begin{array}{r}
\left(I_{h}-\left(I_{h}+B_{h} \delta t\right)^{k}\right)^{-1}\left(I_{h}-\left(I_{h}+A_{h} \delta t\right)^{k}\right) u \\
\approx\left(I_{h}-\left(I_{h}+B_{h} k \delta t+\ldots\right)\right)^{-1}\left(I_{h}-\left(I_{h}+A_{h} k \delta t+\ldots\right)\right) u \approx B_{h}^{-1} A_{h} u
\end{array}
$$

For these modes, the eigenvalues are independent of $k$ and they behave as the eigenvalues of $B_{h}^{-1} A_{h}$, which were discussed in the previous section. However, the behaviour of the rightmost eigenvalues is significantly different. To understand this, we look at the dominant part of $A_{h}$ and $B_{h}$. This is tridiag $\left(1 / h^{2},-2 / h^{2}, 1 / h^{2}\right)$ multiplied with, respectively, $D$ 
and $D^{*}$. The largest eigenvalues of this dominant part is approximated by, respectively, $-4 D / h^{2}$ and $-4 D^{*} / h^{2}$, which leads to the approximation of the dominant eigenvalue of the preconditioned system

$$
\mu^{*}=\frac{\left(1-(1-4 D \nu)^{k}\right)}{\left(1-\left(1-4 D^{*} \nu\right)^{k}\right)} \in \mathbb{R} .
$$

If $D=D^{*}$, then $\mu^{*}=1$ for all values of $k$; however,when $D \neq D^{*}$, this values depends on $k$ and $\nu$, and will therefore not lie on the circle that is found for $k=1$. Only for small $\nu$, we have $\mu^{*} \approx D / D^{*}$.

In a next experiment, we examine the eigenvalues of matrix (3.5) for fixed $\nu=0.2$ and increasing $k$, again considering a central difference discretization of equations (3.1) and (3.2) with $D=1, D^{*}=0.7, \beta=10, \delta=0, \delta^{*}=\delta^{-}$(obtained for $k=1$ ) and $h=0.02$ (corresponding to $\gamma=0.1$ ). The results are shown in figure 5 (right). We see that the leftmost eigenvalues are still well approximated by the values for $k=1$. The circles, however, quickly become perturbed and the value $\mu^{*} \rightarrow 1$; this can be explained with the help of (5.23) by realizing that, for numerical stability of the time integration, both $|1-4 D \nu|<1$ and $\left|1-4 D^{*} \nu\right|<1$. For $k>1$, we also see two real eigenvalues at the left end of the circle, indicating that the value of $\delta^{-}$(for which we have a double real eigenvalue) depends on $k$. We see that the eigenvalues away from the real axis are still well approximated by the values for $k=1$. The circles, however, quickly become perturbed and the value $\mu^{*} \rightarrow 1$; this can be explained by realizing that, for numerical stability of the time integration, both $|1-4 D \nu|<1$ and $\left|1-4 D^{*} \nu\right|<1$.

\subsection{Multiple time steps preconditioned with backward Euler}

In a similar way, we analyze some particular eigenvalues in the spectrum when backward Euler is used as a preconditioner. The advantage of using backward Euler is that one only needs to solve linear systems with the matrix $B_{h}$ to apply the preconditioner [26], reducing the bandwidth compared to $k$ forward Euler steps.

Again, if $u$ is a mode for which $u^{T} A_{h} u \gg u^{T} A_{h}^{2} u$ and $u^{T} B_{h} u \gg u^{T} B_{h}^{2} u$ holds, we make the approximation

$$
\begin{array}{r}
\left(I_{h}-\left(I_{h}-k \delta t B_{h}\right)^{-1}\right)^{-1}\left(I_{h}-\left(I_{h}+A_{h} \delta t\right)^{k}\right) u \\
\approx\left(I_{h}-\left(I_{h}+B_{h} k \delta t+\ldots\right)\right)^{-1}\left(I_{h}-\left(I_{h}+A_{h} k \delta t+\ldots\right)\right) u \approx B_{h}^{-1} A_{h} u .
\end{array}
$$

These modes will behave like the forward Euler case with $k=1$ and align with the circles that were discussed before.

Another particular eigenvalue is found by looking at the dominant parts in $A_{h}$ and $B_{h}$. This eigenvalue is now given by

$$
\frac{1-(1-4 D \nu)^{k}}{1-\left(1+k 4 D^{*} \nu\right)^{-1}}
$$



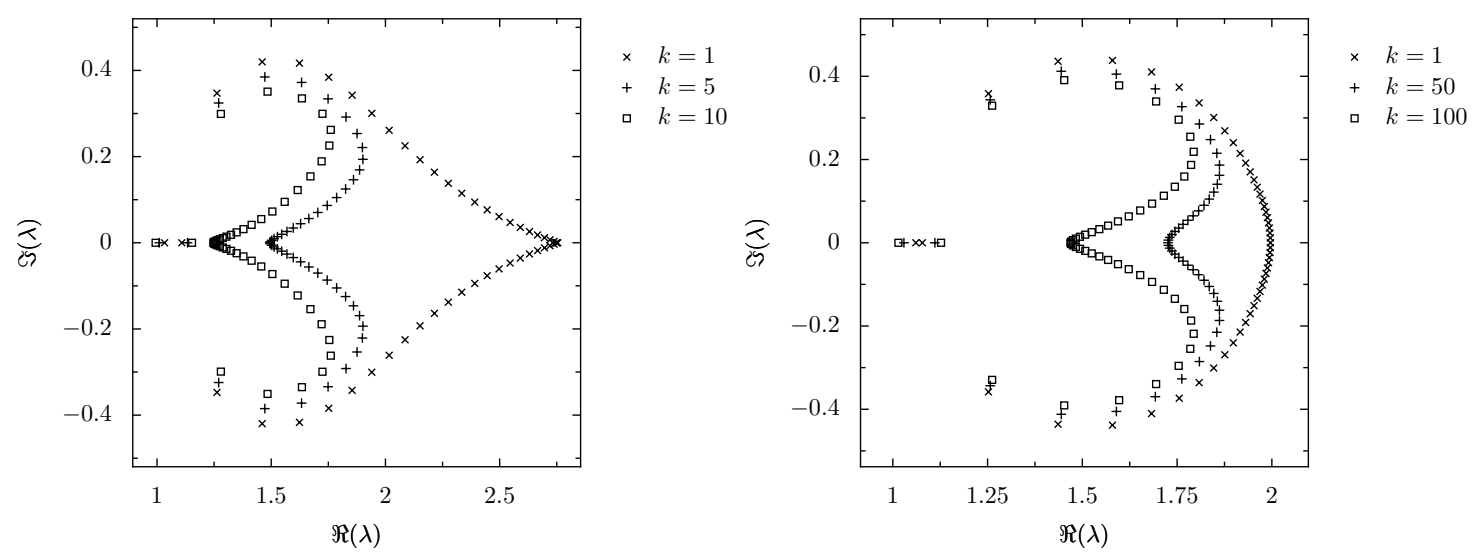

Figure 6: The eigenvalues of the system preconditioned with backward Euler for different values of $k$ for $\nu=0.2$ (left) and $\nu=0.01$ (right).

We illustrate these results by computing the spectrum for $N=49, h=2 \cdot 10^{-2}, D=1$, $\beta=10, \delta=0.3$, using for the preconditioning $D^{*}=0.5$ and $\delta^{*}=\delta^{-}$(obtained for $k=1$ ). Then $\gamma=0.1$. We consider $\nu=0.2$ and $\nu=0.01$, and look at the eigenvalues as a function of $k$. The results are shown in figure 6 .

Again, we see that the leftmost eigenvalues are not significantly different from those for the forward Euler case with $k=1$. We also confirm that for $\nu \ll 1$, the intersection with the real axis is at $\mu^{*}=D / D^{*}$. For $k \rightarrow \infty$, we see $\mu^{*} \rightarrow 1$. Also here, $\delta^{-}$depends on $k$, as is seen from the fact that two real eigenvalues appear for $k>1$.

\section{Numerical convergence tests}

In this section, we illustrate convergence of both Richardson and Krylov iterative methods. In the equation-free context, Richardson iterations are impractical to use, due to the presence of a user-defined damping parameters that depends on the preconditioned spectrum (which is unknown). However, numerical convergence tests using this method are still useful, since they confirm the spectral analysis of section 5, and additionally showing the effects of non-normality. Moreover, these experiments provide an upper bound for the convergence of GMRES. In all experiments, we search the solution $x$ of $A_{h} x=b$, with $b$ a random vector, using $x^{0}=0$ as the initial guess.

\subsection{The case $\beta^{*}=0$.}

We start by considering preconditioning using $\beta^{*}=0$. To this end, we consider the matrix $A_{h}$ with $D=1, \beta=50, \delta=20, h=2 \cdot 10^{-3}$, and $N=499$, leading to $\gamma=0.05$. We define 

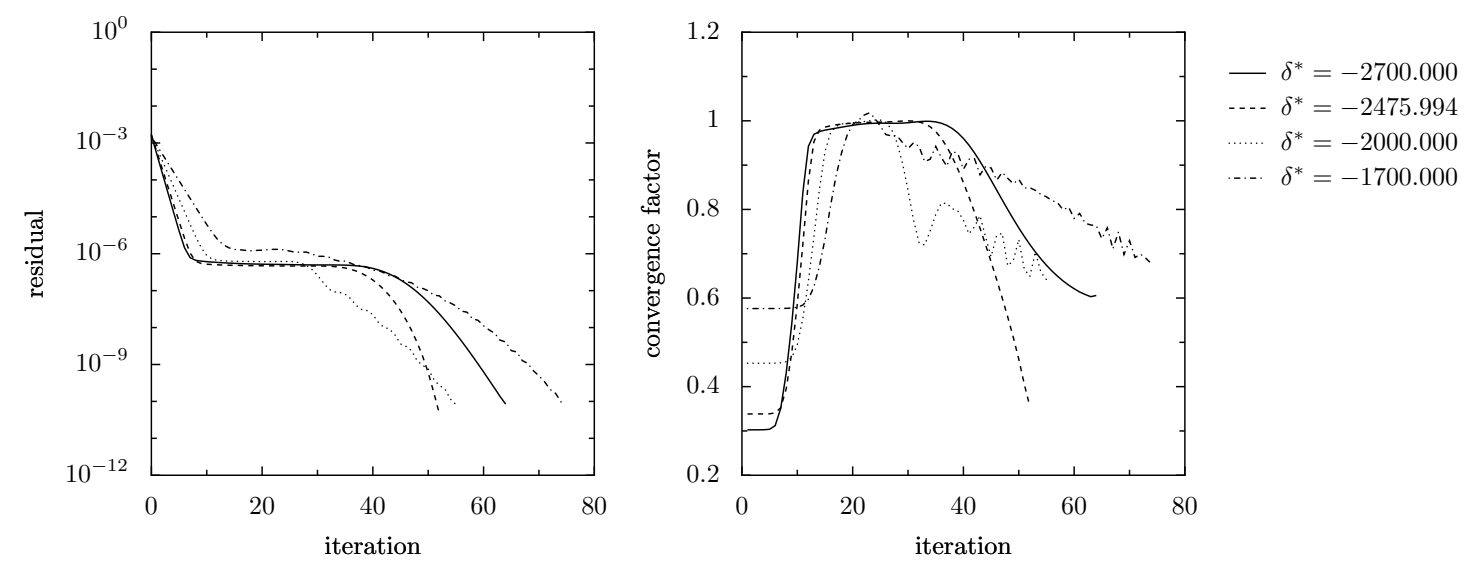

Figure 7: Left: Convergence of Richardson iteration for the example described in section 6.1 with $\beta^{*}=0$ for several values of $\delta^{*}$. Right: Numerical convergence factor as a function of iteration number.

the preconditioner $B_{h}$ using $D^{*}=1$ and $\beta^{*}=0$ using several values of $\delta^{*}$. For each value of $\delta^{*}$, we choose the corresponding optimal Richardson parameter $\tau=1 /\left(\mu_{*}-r\right)$. The results are shown in figure 7 . The figure shows that the convergence factor, computed as the average over 5 iterations, agrees with the spectral analysis during the initial iterations for $\delta^{*}<\delta^{-}$. For $\delta^{*}>\delta^{-}$, although real eigenvalues appear that are not on the derived circle, we still observe initial convergence with a rate that corresponds to the radius of the circle. After a few iterations, we notice stagnation during a number of iterations that is proportional to $\delta^{*}$. This can be explained by the non-normality of the iteration matrix. A careful analysis, however, is somewhat involved [5]. After this stagnation, we again expect convergence with the asymptotic convergence factor. Convergence does accelerate, but the maximal accuracy was reached before we obtained the asymptotic convergence factor. However, convergence accelerates faster when $\delta^{*}$ is closer to $\delta^{-}$. We remark that, for values of $\delta^{*}>\delta^{c} \approx-1237$, Richardson iterations diverge (not shown on figure).

We repeat the experiment using GMRES as the iterative method. The results are shown in figure 8. We see that, in contrast to Richardson iterations, GMRES converges for all values of $\delta^{*}$, and exhibits an initial stagnation period that grows with $\delta^{*}$. Also, the convergence rate initially increases with decreasing $\delta^{*}$, but quickly reaches a rate that is independent of $\delta^{*}$. Again, the tolerance was reached before we could observe the asymptotic convergence factor. 

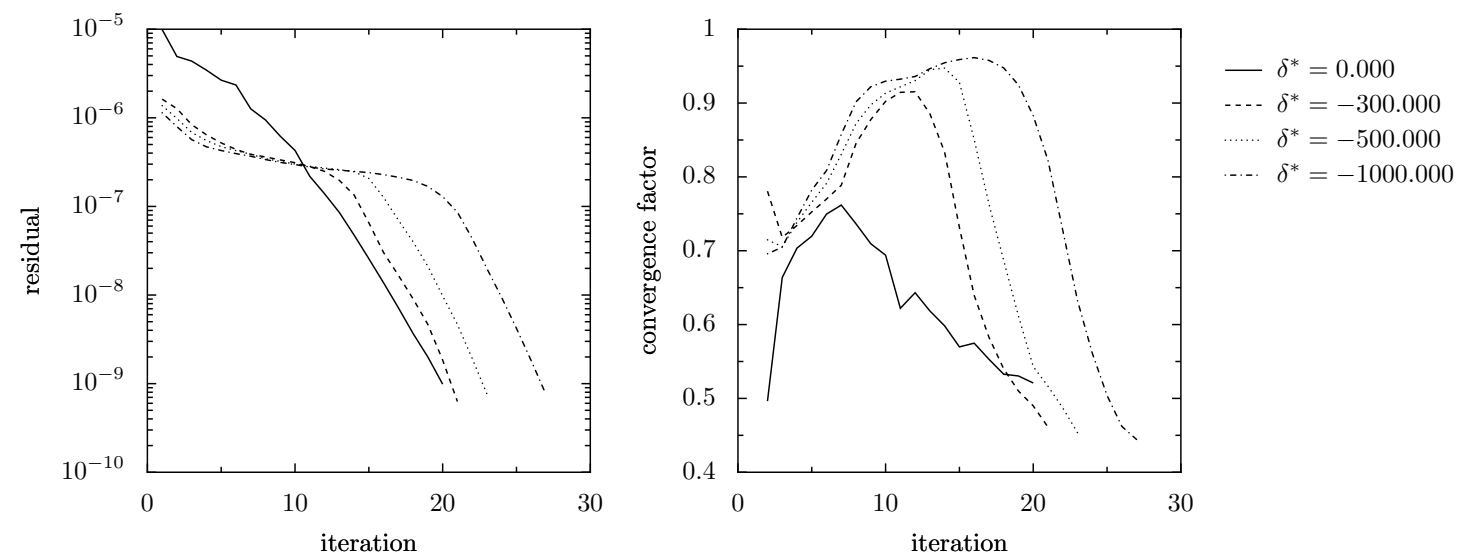

Figure 8: Left: Convergence of GMRES iteration for the example described in section 6.1 with $\beta^{*}=0$ for several values of $\delta^{*}$. Right: Numerical convergence factor as a function of iteration number.

\subsection{The case $\beta^{*} \neq 0$ with $\delta^{*}=\delta^{-}$.}

Since the non-normality is caused by the advection term depending on $\beta$, we now investigate to what extent the above stagnation can be eliminated by adding an advection term to the preconditioner. We again consider the matrix $A_{h}$ with $D=1, \beta=50, \delta=20, h=2 \cdot 10^{-3}$, and $N=499$, leading to $\gamma=0.05$. We define the preconditioner $B_{h}$ using $D^{*}=1$ and several values of $\beta^{*}$, each time using the corresponding value of $\delta^{*}=\delta^{-}\left(\beta^{*}\right)$. We again use Richardson iterations with the corresponding optimal Richardson parameter. The results are shown in figure 9. It can be seen that, as expected, the initial convergence factor is smaller for values of $\gamma^{*}$ closer to $\gamma$. Moreover, the initial convergence factor is the same for $\gamma^{*}=0.03$ and $\gamma^{*}=0.07$, as well as for $\gamma^{*}=0.04$ and $\gamma^{*}=0.06$. We also note that, for $\gamma^{*}<\gamma$, the length of the stagnation period decreases for increasing $\gamma^{*}$. However, we note that there is no stagnation for $\gamma^{*}>\gamma$. We may explain this as follows. From the previous section, we know that stagnation becomes more important for large negative values of $\delta^{-}$. From the observation in example 5.3, it is seen that $\delta^{-}\left(\gamma^{*}\right)$ does not decrease rapidly for $\gamma^{*}>\gamma$, hence we may expect that there will be less stagnation.

We again repeat the experiment using GMRES as the iterative method. The results are shown in figure 10. The conclusions are the same: for values $\gamma^{*}<\gamma$, we have an initial stagnation period with a length that depends on $\left|\gamma^{*}-\gamma\right|$, whereas this stagnation is absent for $\gamma^{*}>\gamma$. 

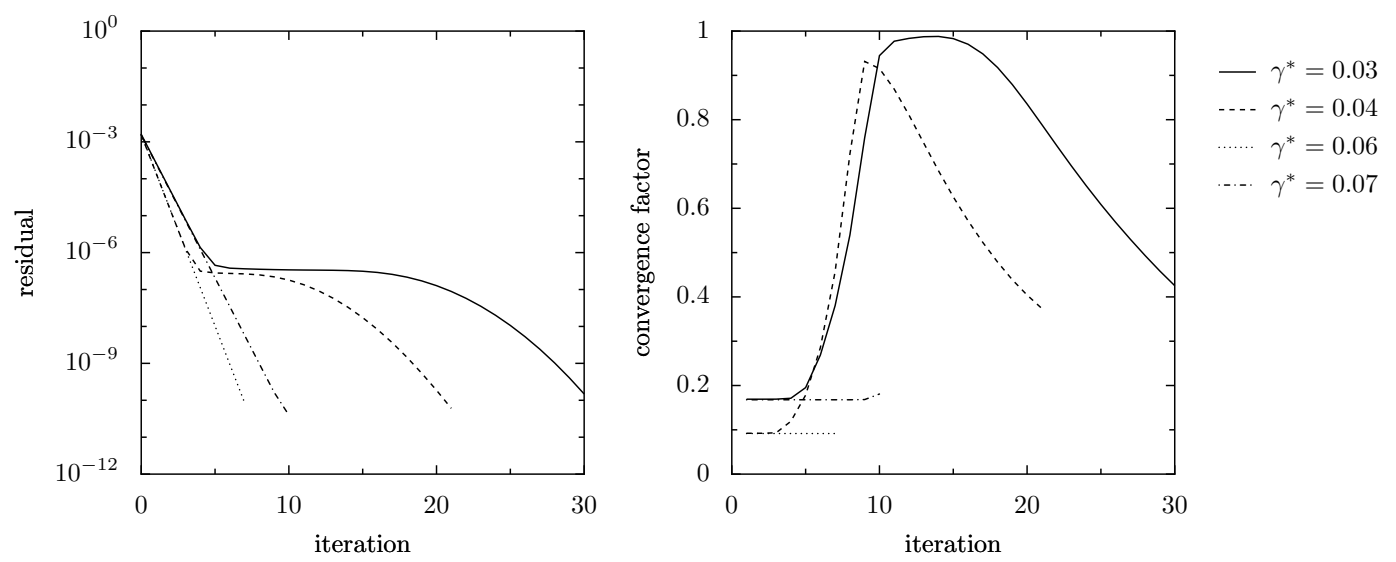

Figure 9: Left: Convergence of Richardson iteration for the example described in section 6.2 for several values of $\beta^{*}$, using $\delta^{*}=\delta^{-}\left(\beta^{*}\right)$. Right: Numerical convergence factor as a function of iteration number.
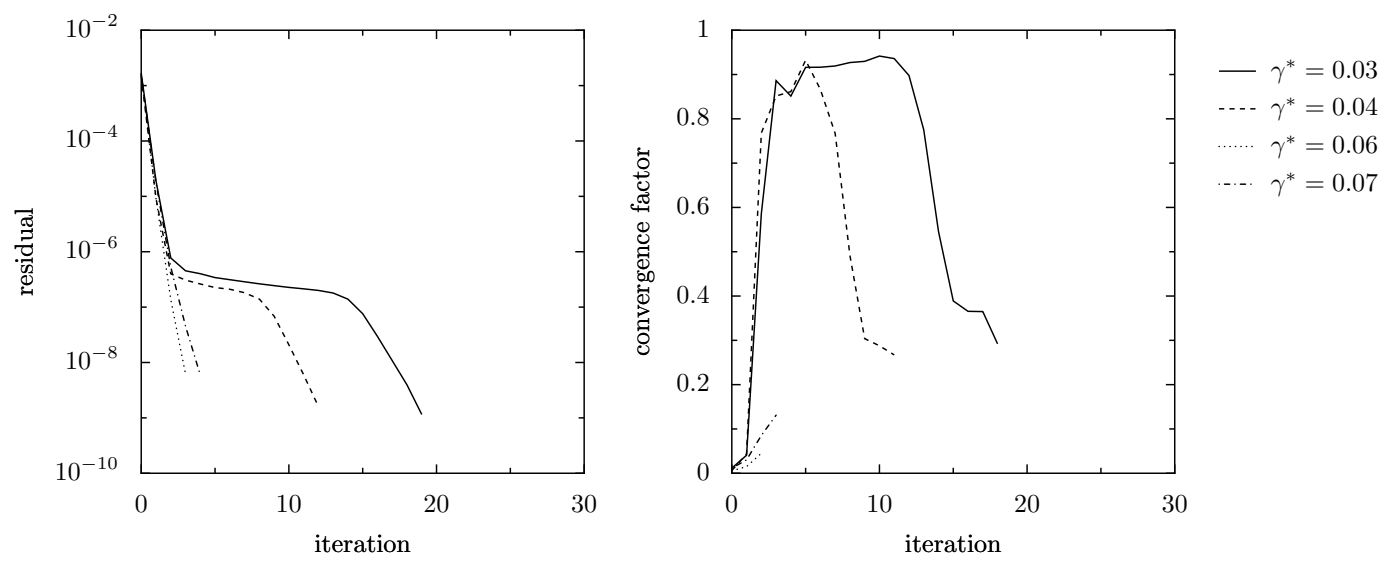

Figure 10: Left: Convergence of GMRES iteration for the example described in section 6.2 for several values of $\beta^{*}$, using $\delta^{*}=\delta^{-}\left(\beta^{*}\right)$. Right: Numerical convergence factor as a function of iteration number. 

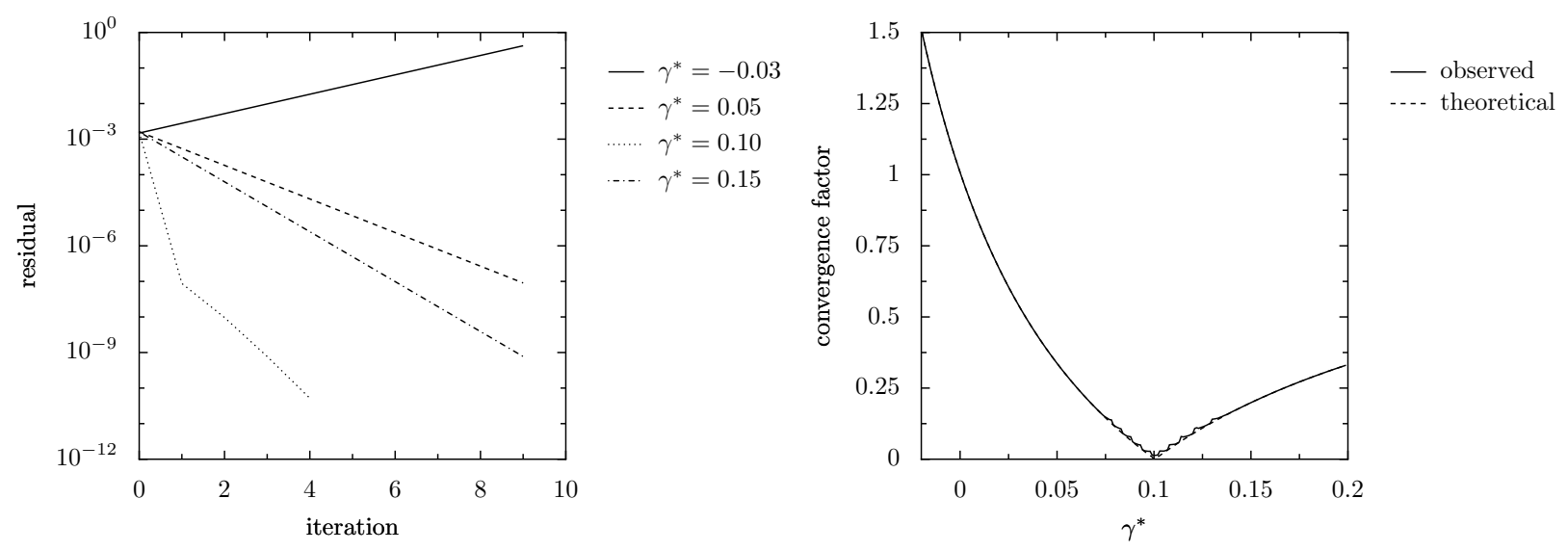

Figure 11: Left: Convergence of Richardson iteration for the example described in section 6.3 for several values of $\beta^{*}$, using $\delta^{*}=0$. Right: Convergence factor as a function of iteration number $\gamma^{*}$, both observed and theoretically.

\subsection{The case $\beta^{*} \neq 0$ with $\delta^{*}=0$.}

Since we are interested in cases where the coefficients in the matrix $A_{h}$ are not known exactly, we cannot expect to have the optimal value $\delta^{-}$available. We now investigate convergence when we simply use $\delta^{*}=0$, as a function of $\gamma^{*}$. We again consider the matrix $A_{h}$ with $D=1, \beta=100, \delta=20, h=2 \cdot 10^{-3}$, and $N=499$, leading to $\gamma=0.1$. We define the preconditioner $B_{h}$ using $D^{*}=1$ and $\delta^{*}=0$ and several values of $\gamma^{*} \in[-0.05,0.2]$, and again use Richardson iterations with the corresponding optimal Richardson parameter. Note that $\delta^{-}>0$ for $\gamma^{*}>0.1$, so that, with $\delta^{*}=0$, not all eigenvalues are on the circle in that case. Figure 11 (left) shows convergence for several values of $\gamma^{*}$. We see that there never is stagnation (which could be expected since $\delta^{*}=0$ ). We also show the convergence factor, which is computed as the 6 -th root of the ratio of the residual at iterations $n$ and $n-6$, with $n$ the total number of iterations at convergence, as a function of $\gamma^{*}$, see figure 11 (right). There is perfect agreement, even for $\gamma^{*}>0.1$, which indicates that the eigenvalues that are not on the circle do not influence the asymptotic convergence rate.

We now repeat the same experiment with GMRES, and compare the behaviour with Richardson. The results are shown in figure 12. We can make two observations. First, for $\gamma>0$, the asympotic convergence factors of Richardson and GMRES are nearly identical. At the left, we see (for $\gamma^{*}=0.15$ ) that GMRES converges faster during the initial iterations, after which convergence proceeds at the same rate as Richardson iteration. This can be explained by recalling that the eigenvalues of the preconditioned system lie on circles with only a few eigenvalues at the leftmost side. Also note that for negative $\gamma^{*}$, the asymptotic convergence rates differ. For $\gamma^{*}=-0.03$, for instance, Richardson diverges, while GMRES 

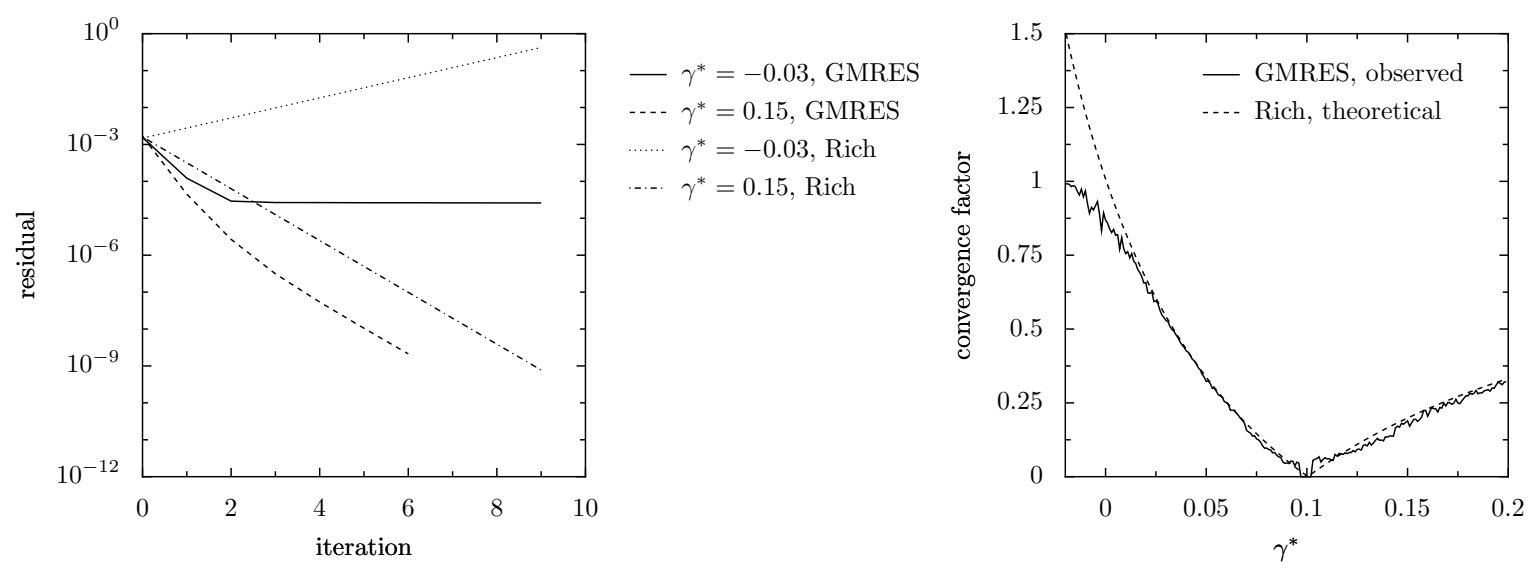

Figure 12: Left: Convergence of Richardson and GMRES iteration for the example described in section 6.3 for several values of $\beta^{*}$, using $\delta^{*}=0$. Right: Convergence factor as a function of iteration number $\gamma^{*}$, both the observed behaviour of GMRES and the theoretical behaviour of Richardson iteration.

converges very slowly $(\rho \approx 1)$, since GMRES cannot diverge.

\subsection{Multiple time steps}

In the next experiments, we assess the influence of taking multiple time-steps. We first consider forward Euler preconditioning leading to preconditioned linear systems of the form (3.5). We consider the matrix $A_{h}$ with $D=1, \beta=50, \delta=20, h=2 \cdot 10^{-3}$, and $N=499$, leading to $\gamma=0.05$. We define the preconditioner $B_{h}$ using $D^{*}=0.7, \beta^{*}=100$ and $\delta^{*}=0$. For the time discretization, we consider $k \in\{1,2,4,8\}$ and $\nu \in[0.01,0.15]$ using forward Euler both in the system and the preconditioner. We again use Richardson iterations with the corresponding optimal Richardson parameter. (As follows from the previous section, the results for GMRES iteration are identical.) The results are shown in figure 13 (left). For $k=1$, the spectrum does not depend on $\nu$, and the observed convergence factor is approximately constant. For $k>1$, the convergence factor decreases with increasing $\nu$, until it reaches a stationary value. This can be explained from the spectral analysis. As $k$ increases, the intersection point $\mu^{*} \rightarrow 1$, and therefore the spectrum gets compacted in a smaller region.

We now repeat the experiment with backward Euler preconditioning. The effect is more dramatic, see figure 13 (right). For each value of $k$, we note a sharp increase of the convergence factor as a function of $\nu$, up to a peak, after which the convergence factor decreases again. The larger the value of $k$, the less pronounced this peak is. (For $k=1$, we even get divergence for $\nu>0.12$.) 

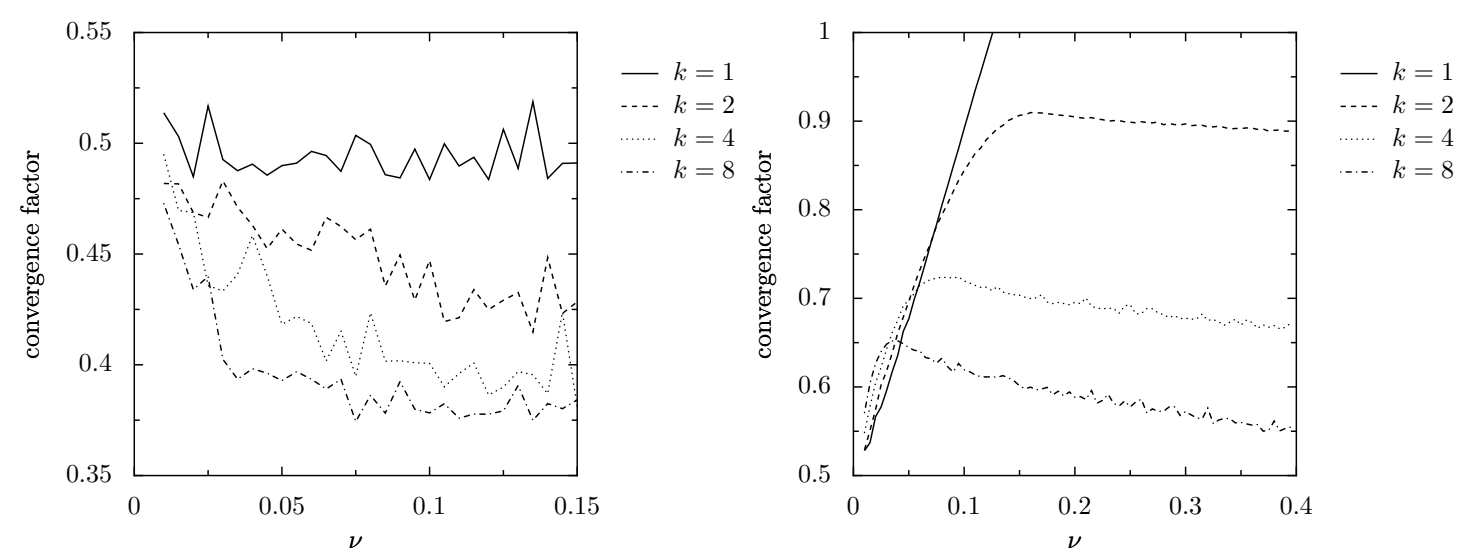

Figure 13: Numerical convergence factor of Richardson iterations for the example described in section 6.4 as a function of $\nu$ for different values of $k$. Left: forward Euler preconditioning. Right: backward Euler preconditioning.

\section{Conclusions and discussion}

The equation-free framework constitutes a class of numerical multiscale methods that allow the computation of solutions of an unavailable coarse-scale model by means of a coarse time-stepper, wrapped around appropriately initialized fine-scale simulations. This coarse time-stepper can be used to estimate matrix-vector products with the system's unavailable Jacobian, allowing the use of iterative methods to directly compute coarse steady states as fixed points of the coarse time-stepper. Since the spectral properties of the time-stepper are unfavorable, preconditioning will be necessary. In this paper, we proposed to precondition equation-free Newton-Krylov computations using an operator of the same type, in which one chooses the parameters to achieve fast convergence.

We have analyzed this equivalent operator preconditioning for a linear reaction-advectiondiffusion $\mathcal{A}$ with constant coefficients, as a function of the parameters of the preconditioner $\mathcal{B}$. The analysis shows that, for given diffusion and advection coefficients $D^{*}$ and $\beta^{*}$ in $\mathcal{B}$, one can always find an optimal reaction coefficient $\delta^{*}=\delta^{-}$, such that the spectrum of the operator $\mathcal{B}^{-1} \mathcal{A}$ is contained in a circle with a radius $r^{-}$that is bounded uniformly in the advection coefficient $\beta$ of the operator $\mathcal{A}$; consequently the Richardson and GMRES convergence rates are also bounded uniformly. (For $\beta^{*}=0$, we recover the result by Manteuffel and Otto [20].) A similar result was obtained for the discrete case, using central differences, provided the Peclet number $\gamma=\beta h /(2 D)<1$, confirming mesh-independent convergence rates. These results were verified numerically. The numerical experiments additionally revealed a stagnation period during convergence, which grows with $\left|\delta^{*}\right|$. Since $\left|\delta^{-}\right|$is does not become very large when $\gamma^{*}>\gamma$, we do not see stagnation in this case.

We also analyzed the effect of time discretization when multiple forward Euler time- 
steps with the operator $\mathcal{A}$ are taken. We considered two cases. When taking the same number of forward Euler time-steps with the preconditioner $\mathcal{B}$, the results show that the convergence rate decreases as $k$ increases. One can, however, also use a single backward Euler step in the preconditioner, which results in a lower bandwidth of the linear systems that need to be solved with the preconditioner. In that case, the convergence slows rapidly as a function of $\nu=\delta t / h^{2}$, and speeds up with $k$.

Choosing $\beta^{*}=0$ and $\delta^{*}=\delta^{-}$results in a symmetric positive definite preconditioner that is easily inverted. However, this choice may result in stagnation of the iterations (especially in one space dimension). Since, in our case, a matrix-vector product involves integration with the coarse time-stepper, we really want to minimize the number of matrixvector products, and we may safely neglect the amount of work that is required for a system solve with the preconditioner. The results then indicate that, to get an acceptable convergence rate, it is advisable to add some advection to the preconditioner, and it is better to overestimate the advection term than to underestimate it. Then, one can simply put the reaction term $\delta^{*}=0$.

This paper only contains an analysis for linear scalar problems with constant coefficients. For systems of coupled nonlinear equations, we propose to use a decoupled system of reaction-advection-diffusion equations as preconditioner. A detailed numerical study of the convergence behaviour for this case is given [25], where we also apply the method to compute coarse traveling waves in a multispeed lattice Boltzmann model for the ionization of gases.

\section{Acknowledgements}

The authors thank Daan Huybrechs, Yannis Kevrekidis, Karl Meerbergen and Eli Turkel for interesting discussions about various aspects of this work. GS is a Postdoctoral Fellow of the Research Foundation - Flanders (FWO - Vlaanderen). This work was supported by the Research Foundation - Flanders through Research Projects G.0130.03 and G.0170.08 and by the Interuniversity Attraction Poles Programme of the Belgian Science Policy Office through grant IUAP/V/22 (GS). The scientific responsibility rests with its authors.

\section{References}

[1] O. Axelsson and J. Karatson, Mesh independent superlinear PCG rates via compact-equivalent operators, SIAM J. Numer. Anal., 45 (2007), pp. 1495-1516.

[2] P. Concus And G.H. Golub, Use of fast direct methods for the efficient numerical solution of nonseparable elliptic equations, SIAM J. Numer. Anal., 10 (1973), pp. 11031120. 
[3] E.G. D'YAKANov, On an iterative method for the solution of a system of finitedifference equations, Dokl. Akad. Nauk, 138 (1961), pp. 522-525.

[4] H.C. Elman and M.H. Schultz, Preconditioning by fast direct methods for nonselfadjoint nonseparable elliptic equations, SIAM J. Numer. Anal., 23 (1986), pp. 44-57.

[5] M Embree, How descriptive are gmres convergence bounds?, Numerical Analysis Report 99/08, Oxford University Computing Laboratory, June 1999.

[6] R. Erban, I.G. Kevrekidis, and H. Othmer, An equation-free computational approach for extracting population-level behavior from individual-based models of biological dispersal, Physica D: Nonlinear Phenomena, 215 (2006), pp. 1-24.

[7] YA Erlangga, CW Oosterlee, and C. Vuik, A Novel Multigrid Based Preconditioner For Heterogeneous Helmholtz Problems, SIAM Journal on Scientific Computing, 27 (2006), pp. 1471-1492.

[8] V. Faber, T. Manteuffel, and S. Parter, On the theory of equivalent operators and application to the numerical solution of uniformly elliptic partial-differential equations, Advances in applied mathematics, 11 (1990), pp. 109-163.

[9] C.W. Gear, T.J. Kaper, I.G. Kevrekidis, And A. Zagaris, Projecting to a slow manifold: Singularly perturbed systems and legacy codes, SIAM Journal on Applied Dynamical Systems, 4 (2005), pp. 711-732.

[10] C.W. Gear and I.G. Kevrekidis, Constraint-defined manifolds: a legacy code approach to low-dimensional computation, Journal of Scientific Computing, 25 (2005), pp. $17-28$.

[11] C. Goldstein, T. Manteuffel, And S. Parter, Preconditioning and boundary conditions without h-2 estimates, l(2) condition numbers and the distribution of the singular values, SIAM J. Numer. Anal., 30 (1993), pp. 343-376.

[12] J.E. GunN, The solution of elliptic difference equations by semi-explicit iterative techniques, Numerische Mathematik, 6 (1964), pp. 181-184.

[13] I.G. Kevrekidis, C.W. Gear, J.M. Hyman, P.G. Kevrekidis, O. Runborg, AND C. Theodoropoulos, Equation-free, coarse-grained multiscale computation: enabling microscopic simulators to perform system-level tasks, Comm. Math. Sciences, 1 (2003), pp. $715-762$.

[14] I.G. KevreKIDIS AND G. SAmaey, Equation-free multiscale computation: algorithms and applications, Annual Review on Physical Chemistry, 60 (2009). In press. 
[15] D.A. Knoll And D.E. Keyes, Jacobian-free Newton-Krylov methods: a survey of approaches and applications, Journal of Computational Physics, 193 (2004), pp. 357397.

[16] Tino Koch And Jörg Liesen, The conformal 'bratwurst' maps and associated Faber polynomials, Numerische Mathematik, 86 (2000), pp. 173-191.

[17] Chao Li, Ute Ebert, W. J. M Brok, and Willem Hundsdorfer, Spatial coupling of particle and fluid models for streamers: where nonlocality matters, J Phys D Appl Phys, 41 (2008), p. 032005.

[18] K. Lust, Numerical bifurcation analysis of periodic solutions of partial differential equations, PhD thesis, Katholieke Universiteit Leuven, 1997.

[19] K. Lust, D. Roose, A. Spence, And A.R. Champneys, An adaptive NewtonPicard algorithm with subspace iteration for computing periodic solutions, SIAM Journal on Scientific Computing, 19 (1998), pp. 1188-1209.

[20] T. Manteuffel And J. Otтo, Optimal equivalent preconditioners, SIAM J. Numer. Anal., 30 (1993), pp. 790-812.

[21] T. Manteuffel And S. Parter, Preconditioning and boundary conditions, SIAM J. Numer. Anal., 27 (1990), pp. 656-694.

[22] L. Qiao, R. Erban, C.T. Kelley, and I.G. Kevrekidis, Spatially distributed stochastic systems: equation-free and equation-assisted bifurcation analysis, Journal of Chemical Physics, 125 (2006), p. 204108.

[23] Y. SAAD, Iterative Methods for Sparse Linear Systems, Society for Industrial and Applied Mathematics, Philadelphia, PA, USA, 2003.

[24] Y. SAAD AND M.H. Schultz, GMRES: A generalized minimal residual algorithm for solving nonsymmetric linear systems, SIAM Journal of Scientific and Statistical Computing, 7 (1986), pp. 856-869.

[25] G. Samaey, W. Vanroose, and I.G. Kevrekidis, Equivalent operator preconditioning for equation-free Newton-Krylov computations. in preparation, 2009.

[26] G. Samaey, W. Vanroose, D. Roose, and I.G. Kevrekidis, Newton-Krylov solvers for the equation-free computation of coarse traveling waves, Computer Methods in Applied Mechanics and Engineering, (2008). In press.

[27] G.M. Schroff And H.B. Keller, Stabilization of unstable procedures: the recursive projection method, SIAM Journal on Numerical Analysis, 30 (1993), pp. 1099-1120. 
[28] J. Simoens and S. Vandewalle, Waveform Relaxation with Fast Direct Methods as Preconditioner, SIAM Journal on Scientific Computing, 21 (2000), pp. 1755-1773.

[29] C. Theodoropoulos, Y.H. Qian, And I.G. Kevrekidis, Coarse stability and bifurcation analysis using time-steppers: a reaction-diffusion example, in Proc. Natl. Acad. Sci., vol. 97, 2000, pp. 9840-9845.

[30] P. Van Leemput, W. Vanroose, and D. Roose, Mesoscale analysis of the equationfree constrained runs initialization scheme, Multiscale Modeling and Simulation, 6 (2007), pp. 1234-1255.

[31] O. Widlund, A Lanczos method for a class of non-symmetric systems of linear equations, SIAM J. Numer. Anal., 15 (1978), pp. 801-812. 